\title{
Analytically calibrated Box-Cox percentile limits for duration and event-time models
}

\author{
Zhenlin Yang $^{a, 1}$, Albert K. Tsui ${ }^{b}$ \\ ${ }^{a}$ School of Economics and Social Sciences, Singapore Management University \\ 469 Bukit Timah Road, Singapore 259-756 \\ ${ }^{b}$ Department of Economics, National University of Singapore \\ 1 Arts Link, Singapore 117570
}

\begin{abstract}
This paper proposes a unified approach to constructing confidence limits for a future percentile duration or event-time. The construction is based on an analytical calibration of the Box-Cox type "plug-in" percentile limits (PL). The performance of the calibrated Box-Cox PL is investigated using Monte Carlo experiments. Comparisons are made with PLs that are specifically designed for a particular distribution such as Weibull and lognormal. Excellent performances of the calibrated Box-Cox PL are observed. Simulation based on other popular duration models such as gamma and inverse Gaussian reveal that the proposed PL is robust against distributional assumptions and that it performs much better than the distribution-free PL. An empirical illustration is also provided.
\end{abstract}

JEL Classification: C15; C41

Subj. Class.: IM10

Keywords: Analytical calibration; Box-Cox transformation; Duration model; Event-time model; Percentile limits

\footnotetext{
${ }^{1}$ Corresponding author.

E-mail addresses: zlyang@smu.edu.sg (Z. L. Yang)
} 


\section{Introduction}

Duration analysis has become a rapidly growing area of econometric research in the past two decades. Kieffer (1988) gives a detailed survey on the economic duration and hazard models. Indeed, actual and potential areas of applications include the strike duration (Lancaster, 1972; Kennan, 1985), unemployment duration (Lancaster, 1979; Sider, 1985; Kiefer, et al., 1985), financial transaction duration (Engle and Russell, 1998; Zhang, et al., 2001), duration of marriages, time to adoption of new technology, lifetimes of firms, product durability, and others ${ }^{2}$. Essentially, the literature of economic duration analysis is heavily based on statistical methods developed in areas of industrial engineering and biomedical sciences, concentrating on model specification and analysis of duration data. The issue of adequately predicting future percentile durations has remained relatively unexplored. See Collins (1991) and Yang (1999b, 2001).

In this paper, we propose a unified approach to constructing confidence limits for the percentile of a future duration or event-time. Percentile durations can offer important scopes for empirical studies in applied economics. For example, if one is concerned with the 'typical' unemployment duration, then the median duration (the 50th percentile) is appropriate. If one wants to know when only $1 \%$ of the firms turn bankrupt, then the 1st percentile life is essential. And if one wants to gauge the duration after which most of the unemployed find a job, then the 95th or even 99th percentile are useful. However, the existing methods to construct reliable confidence limits for future percentiles still face some practical problems. First, it is often unclear which lifetime distribution should be used. Second, even if one picks the correct model, the percentile limits for this model could be unavailable, unsatisfactory, or too complicated to be implemented in practice. Third, the standard nonparametric or distribution-free methods do not give reliable percentile limits for extreme percentiles as such methods often require unrealistically large samples. Hence, it is highly desirable to construct a unified PL for a percentile duration of event-time that works for any duration or event-time model, thereby reducing the impact of distributional

\footnotetext{
${ }^{2}$ More useful references include Heckman and Singer (1984a, b), Kiefer (1988), Lancaster (1985, 1990), Ryu (1993), Torelli and Trivellato (1993), Koop and Ruhm (1993), Saha and Hilton (1997), and Baker and Melino (2000)
} 
assumptions in econometric models. See Heckman and Singer (1984b).

The Box-Cox transformation (Box and Cox, 1964) aims to transform non-negative continuous observations to exact or near normality. Hernandze and Johnson (1980) showed that observations following popular distributions such as lognormal, Weibull, gamma, and inverse Gaussian are all transformable to exact or near normality by the Box-Cox power transformation. Moreover, percentiles are invariant under a one-to-one transformation. As such, we shall use the Box-Cox method to construct percentile limits for a transformed percentile based on normal theory, and then retrieve percentile limits for the original percentile from a simple inversion. The whole process is valid if the transformation is known and exact normality is achievable. However, the problem is that the transformation (parameter) is often unknown and has to be estimated from the data. In this case, a common practice is to replace the unknown transformation by its estimate which is then treated as if it were the true one (Box and Cox, 1964; Collins, 1991; Hahn and Meeker, 1991). Clearly, this approach ignores the effect of estimating transformation. If such an approach is successful, the standard methods of statistical inference become applicable and it renders a unified approach to constructing confidence limits for percentile durations. However, as shown in Yang (2001) for the case of median and as we will show in this paper for the case of general percentile, the effect of estimating transformation cannot be ignored and a correction to the confidence limits is necessary.

In this paper, we extend the approach of Yang (2001) to derive the analytical calibration of the Box-Cox percentile limits (PL). As the construction of normal-theory PL involves a noncentral $t$ distribution, the analytical calibration now comes with two factors, one corresponding to the variance adjustment and the other the mean adjustment. However, the two calibration factors remain simple, and Monte Carlo experiments show that the calibrated PL is accurate and robust against the distributional assumptions. The rest of this paper is set out as follows. Section 2 presents the Box-Cox PL and its asymptotic properties. Section 3 derives the analytical calibration of the Box-Cox PL. Section 4 presents Monte Carlo simulations for checking the small sample performance of the Box-Cox and calibrated Box-Cox PLs. Comparisons are also made with the existing PLs that are specifically designed for a certain distribution as well as the distribution-free PL. Section 
5 illustrates the superiority of the proposed PLs using the strike duration data of Kennan (1985). Finally Section 6 gives some concluding remarks. All proofs are contained in the Appendix.

\section{Box-Cox percentile limits and their properties}

Let $\mathbf{Y}=\left(Y_{1}, Y_{2}, \cdots, Y_{n}\right)$ be a sample of $n$ non-negative durations or event-times from a certain distribution. Let $Y_{0}$ be a future observation from the same population. We are interested in constructing the confidence limits for $y_{p}$, the $100 p$ th percentile of $Y_{0}$. Suppose that there exists a monotonic increasing transformation $h(\cdot, \lambda)$, indexed by a transformation parameter $\lambda$, such that the transformed observations are normally distributed with mean $\mu$ and standard deviation $\sigma$. That is,

$$
h\left(Y_{i}, \lambda\right) \stackrel{i i d}{\sim} N\left(\mu, \sigma^{2}\right), i=1, \cdots, n
$$

It can be shown that the maximum likelihood estimators (MLE) of $\mu, \sigma$ and $\lambda$ are

$$
\begin{aligned}
\hat{\mu}(\hat{\lambda}) & =\frac{1}{n} 1_{n}^{\prime} h(\mathbf{Y}, \hat{\lambda}) \equiv \bar{h}(\mathbf{Y}, \hat{\lambda}) \\
\hat{\sigma}(\hat{\lambda}) & =\frac{1}{\sqrt{n}}\left\|h(\mathbf{Y}, \hat{\lambda})-\bar{h}(\mathbf{Y}, \hat{\lambda}) 1_{n}\right\| \\
\hat{\lambda} & =\arg \min _{\ell} \frac{\left\|h(\mathbf{Y}, \ell)-\bar{h}(\mathbf{Y}, \ell) 1_{n}\right\|}{\dot{h}_{y}(\mathbf{Y}, \ell)}
\end{aligned}
$$

where $\|\cdot\|$ is the Euclidian norm, $1_{n}$ is a vector of $1^{\prime} s$, and $\dot{h}_{y}(\mathbf{Y}, \ell)$ is the geometric mean of $\left\{h_{y}\left(Y_{i}, \ell\right)=\partial h\left(Y_{i}, \ell\right) / \partial Y_{i}, i=1, \cdots, n\right\}$. When $\lambda$ is known, the restricted MLEs of $\mu$ and $\sigma$ are denoted by $\hat{\mu}(\lambda)$ and $\hat{\sigma}(\lambda)$, respectively.

When $\lambda$ is known, the usual normal theories can directly be applied to give a prediction or confidence interval for the transformed quantity of interest, which is then inverted to give prediction or confidence interval of the quantity in the original scale. However, when $\lambda$ is unknown, the usual approach is to follow Box and Cox (1964) to estimate $\lambda$ and then proceed with the usual inferences as if the estimated transformation parameter $\lambda$ were the true one. This is the popular Box-Cox Transformation Method. 


\subsection{The Box-Cox percentile limits}

As $h$ is an one-to-one mapping, $y_{p}$ is related to $z_{p}$, the $100 p$ th percentile of the standard normal, via $h\left(y_{p}, \lambda\right)=\mu+\sigma z_{p}$. A natural estimator of $h\left(y_{p}, \lambda\right)$ is thus $\hat{\mu}(\lambda)+s(\lambda) z_{p}$, and a natural pivotal quantity for making inference about $y_{p}$ is

$$
T_{p}(\lambda)=\frac{\hat{\mu}(\lambda)+s(\lambda) z_{p}-h\left(y_{p}, \lambda\right)}{s(\lambda) / \sqrt{n}},
$$

where $s(\lambda)$ is the sample standard deviation in $\lambda$-scale. Rewriting $T_{p}(\lambda)$ as

$$
T_{p}(\lambda)=\frac{\sqrt{n}[\hat{\mu}(\lambda)-\mu] / \sigma-\sqrt{n} z_{p}}{s(\lambda) / \sigma}+\sqrt{n} z_{p}
$$

one immediately sees that the first term on the right hand side of $(2)$ is $t_{n-1}\left(-\sqrt{n} z_{p}\right)$, a noncentral $t$ random variable with $n-1$ degrees of freedom and a noncentrality parameter $-\sqrt{n} z_{p}$. This implies that $T_{p}(\lambda) \sim t_{n-1}\left(-\sqrt{n} z_{p}\right)+\sqrt{n} z_{p}$. Hence,

$$
\mu_{T}=E\left[T_{p}(\lambda)\right]=-\sqrt{n} z_{p}\left[\sqrt{\frac{n-1}{2}} \frac{\Gamma\left(\frac{n-2}{2}\right)}{\Gamma\left(\frac{n-1}{2}\right)}-1\right],
$$

and

$$
\sigma_{T}^{2}=\operatorname{Var}\left[T_{p}(\lambda)\right]=\frac{n-1}{n-3}\left(1+n z_{p}^{2}\right)-n z_{p}^{2} \frac{n-1}{2}\left[\frac{\Gamma\left(\frac{n-2}{2}\right)}{\Gamma\left(\frac{n-1}{2}\right)}\right]^{2} .
$$

It can be shown that, as $n$ becomes large, $\mu_{T}$ converges to 0 and $\sigma_{T}$ converges to 1. However, the convergence of $\sigma_{T}$ can be very slow for small or large percentiles. This implies that when $n$ is small to moderate, $\mu_{T}$ is close to 0 but $\sigma_{T}$ can be significantly larger than 1 . These observations are useful in developing the calibration factors for the percentile limits when $\lambda$ is unknown, which will be discussed in Section 3.

Denote the $\delta$ th quantile of $t_{n-1}\left(-\sqrt{n} z_{p}\right)$ by $t_{n-1}^{\delta}\left(-\sqrt{n} z_{p}\right)$. Then, with an exact probability of $(1-\alpha)$ the following will be true

$$
t_{n-1}^{\alpha / 2}\left(-\sqrt{n} z_{p}\right)+\sqrt{n} z_{p} \leq T_{p}(\lambda) \leq t_{n-1}^{1-\alpha / 2}\left(-\sqrt{n} z_{p}\right)+\sqrt{n} z_{p},
$$

which leads immediately to the exact $100(1-\alpha) \%$ confidence limits for $h\left(y_{p}, \lambda\right)$

$$
\left\{\hat{\mu}(\lambda)-t_{n-1}^{1-\alpha / 2}\left(-\sqrt{n} z_{p}\right) \frac{s(\lambda)}{\sqrt{n}}, \hat{\mu}(\lambda)-t_{n-1}^{\alpha / 2}\left(-\sqrt{n} z_{p}\right) \frac{s(\lambda)}{\sqrt{n}}\right\}
$$

Finally, inverting the lower limit $L(\lambda)$ and the upper limit $U(\lambda)$ of the interval in (5), we obtain the exact $100(1-\alpha) \%$ confidence limits for $y_{p}$ as follows.

$$
\left\{h^{-1}[L(\lambda), \lambda], h^{-1}[U(\lambda), \lambda]\right\}
$$


where $h^{-1}$ represents the inverse transformation.

When the transformation parameter $\lambda$ is unknown, a common practice is to replace the unknown $\lambda$ by its MLE $\hat{\lambda}$, and treat the estimated transformation as the true one. ${ }^{3}$ The resultant confidence limits for $y_{p}$ are

$$
\left\{h^{-1}[L(\hat{\lambda}), \hat{\lambda}], h^{-1}[U(\hat{\lambda}), \hat{\lambda}]\right\}
$$

As the confidence limits given in (7) are obtained by directly plugging $\hat{\lambda}$ in (6), they are termed in this paper as the Box-Cox Percentile Limits. Note that the Box-Cox percentile limits ignore the possible effect of the transformation estimation. Carroll and Ruppert (1981) showed that such an effect is usually small for the case of median. In contrast, we show that the effect of estimating transformation can be very large when extreme percentiles are concerned. For most percentiles (10\% to $90 \%$, say), the effect is usually small, but not negligible.

\subsection{Large sample properties of Box-Cox PL}

We say that effect of estimating transformation is small if the PL defined in (7) behaves like the PLs defined in (6), i.e., the coverage probabilities and the expected lengths of the two PLs are similar. Clearly, for this to be true, it is necessary that

$$
T_{p}(\hat{\lambda})=\frac{\hat{\mu}(\hat{\lambda})+s(\hat{\lambda}) z_{p}-h\left(y_{p}, \hat{\lambda}\right)}{s(\hat{\lambda}) / \sqrt{n}}
$$

behaves like $T_{p}(\lambda)$ as expressed in (1). Unfortunately, it is not the case as shown by Theorem 1 below. We first state a lemma with its proof given in the Appendix.

Lemma 1 Under the usual regularity conditions for the likelihood inference theory, we have that $\sqrt{n}(\hat{\lambda}-\lambda)$ and $T_{p}(\lambda)$ are asymptotically independent.

Theorem 1. Assume that i) $\hat{\lambda}$ is root-n consistent, and ii) both $E\left[h_{\lambda}\left(Y_{i}, \lambda\right)\right]$ and $E\left[h\left(Y_{i}, \lambda\right) h_{\lambda}\left(Y_{i}, \lambda\right)\right]$ exist, where $h_{\lambda}\left(Y_{i}, \lambda\right)=\frac{\partial}{\partial \lambda} h\left(Y_{i}, \lambda\right)$. Then, we have

$$
T_{p}(\hat{\lambda})=T_{p}(\lambda)+\frac{\sqrt{n}(\hat{\lambda}-\lambda)}{\sigma}\left\{E\left[h_{\lambda}\left(Y_{0}, \lambda\right)\right]+E\left[s_{\lambda}(\lambda)\right] z_{p}-h_{\lambda}\left(y_{p}, \lambda\right)\right\}+O_{p}\left(n^{-1 / 2}\right),
$$

\footnotetext{
${ }^{3}$ See also Collins (1991), Hahn and Meeker (1991, p.72), and Yang (1999b, 2001)
} 
where $s_{\lambda}(\lambda)=\frac{d}{d \lambda} s(\lambda)$. It follows that

$$
\begin{aligned}
E\left[T_{p}(\hat{\lambda})\right] & =E\left[T_{p}(\lambda)\right]+O\left(n^{-1 / 2}\right), \\
\operatorname{Var}\left[T_{p}(\hat{\lambda})\right] & =\operatorname{Var}\left[T_{p}(\lambda)\right]+c^{2}(\mu, \sigma, \lambda)+O\left(n^{-1 / 2}\right),
\end{aligned}
$$

where $c^{2}(\mu, \sigma, \lambda)=\operatorname{Var}\left[\frac{\sqrt{n}(\hat{\lambda}-\lambda)}{\sigma}\right]\left[E h_{\lambda}\left(Y_{0}, \lambda\right)+E s_{\lambda}(\lambda) z_{p}-h_{\lambda}\left(y_{p}, \lambda\right)\right]^{2}$.

Proof of Theorem 1 is given in the Appendix. Note that the order of the second term on the right hand side of (9) is $O_{p}(1)$, which is of the same order as $T_{p}(\lambda)$. This means that $T_{p}(\hat{\lambda})$ and $T_{p}(\lambda)$ do not agree in distribution even when $n$ is large. The results in (10) and (11) imply that the difference is mainly in the second moments. Hence, a correction to the variance of $T_{p}(\hat{\lambda})$ is necessary. The feasibility of obtaining an analytical correction depends on the availability of an explicit expression of $c^{2}(\mu, \sigma, \lambda)$, which will be discussed in the next section.

\section{Analytically calibrated Box-Cox PL}

We now derive an analytical adjustment to $T_{p}(\hat{\lambda})$ so that its limiting distribution is equivalent to that of $T_{p}(\lambda)$, which in turn gives a corresponding adjustment to the BoxCox PL. From the results of Theorem 1 we see that $E\left[T_{p}(\hat{\lambda})\right]$ agrees with $E\left[T_{p}(\lambda)\right]$ when $n$ is large, but $\operatorname{Var}\left[T_{p}(\hat{\lambda})\right]$ exceeds $\operatorname{Var}\left[T_{p}(\lambda)\right]$ by a factor $c^{2}(\mu, \sigma, \lambda)$, called the variance inflation factor. To rectify the discrepancies in variances, it is necessary to obtain a convenient estimator of $c^{2}(\mu, \sigma, \lambda)$. Clearly, an explicit expression for $c^{2}(\mu, \sigma, \lambda)$ renders a very simple estimator.

\subsection{The power transformation}

In general, the results obtained above are valid for any monotonic increasing transformation. However, to derive an explicit expression or approximation to $c^{2}(\mu, \sigma, \lambda)$, it is necessary to specify the transformation function. There are several options available. The most appropriate one for transforming duration and event-time data may be the Box-Cox 
power transformation

$$
h\left(Y_{i}, \lambda\right)= \begin{cases}\left(Y_{i}^{\lambda}-1\right) / \lambda, & \lambda \neq 0, \\ \log Y_{i}, & \lambda=0,\end{cases}
$$

Some remarks are in order: i) $h_{y}\left(Y_{0}, \lambda\right)=Y_{0}^{\lambda-1}$; ii) $h_{\lambda}\left(Y_{0}, \lambda\right)=\frac{1}{\lambda}\left[Y_{0}^{\lambda} \log Y_{0}-h\left(Y_{0}, \lambda\right)\right]$, which becomes $\frac{1}{2} \log ^{2} Y_{0}$ when $\lambda=0$; iii) for $h\left(y_{p}, \lambda\right)=\mu+\sigma z_{p}$, we have $y_{p}=\left[1+\lambda\left(\mu+\sigma z_{p}\right)\right]^{1 / \lambda}$, which becomes $\exp \left(\mu+\sigma z_{p}\right)$ when $\lambda=0$; and iv) $h\left(Y_{i}, \lambda\right)$ cannot be exact normal unless $\lambda=0$. It, however, can be very close to normal if $\lambda \sigma /(1+\lambda \mu)$ is small. See Yang (1999a) for a detailed discussion on this issue.

Corollary 1. Under the conditions of Theorem 1, the standard Box-Cox power transformation function, and $\theta=\lambda \sigma /(1+\lambda \mu)$, we have for large $n$,

$$
\begin{aligned}
& \text { (i) } c^{2}(\mu, \sigma, \lambda) \approx \frac{1}{6}\left(1-z_{p}^{2}-3 \theta z_{p}+\theta z_{p}^{3}\right)^{2} \text {, when } \lambda \neq 0, \theta \ll 1, \\
& \text { (ii) } c^{2}(\mu, \sigma, \lambda)=\frac{1}{6}\left(1-z_{p}^{2}\right)^{2}, \text { when } \lambda=0 .
\end{aligned}
$$

The proof of Corollary 1 is given in the Appendix. It turns out the variance inflation factor has very simple expressions. It depends on the parameters only through $\theta$ when $\lambda \neq 0$, and is parameter free when $\lambda=0$. When $p=0.5$ it reduces to a pure constant $1 / 6$, which is the special case of median considered in Yang (2001).

The results of Corollary 1 allow us to draw some qualitative conclusions regarding the magnitude of variance inflation due to transformation estimation. Note that $\theta$ is assumed to be small. Thus, the magnitude of the variance inflation $c^{2}(\mu, \sigma, \lambda)$ depends mainly on $z_{p}$. It is small when $z_{p}^{2}$ is close to 1 (i.e., around 16 th or 84 th percentiles); not negligible when $z_{p}^{2}$ is close to zero (i.e., around the median); and large when $z_{p}^{2}$ is much larger than 1 (i.e., at the extreme percentiles). We note in passing that these features provide important practical implications as to when the more conventional Box-Cox Pl works well and when it needs an adjustment.

\subsection{Analytical calibration}

We are now ready to discuss calibrating the Box-Cox PL so that it has correct or approximately correct coverage for any percentile. Write $c^{2}(\mu, \sigma, \lambda)$ as $c^{2}(\theta)$ and define 
$\hat{\theta}=\hat{\lambda} \hat{\sigma} /(1+\hat{\lambda} \hat{\mu})$. From Theorem 1, we see that the adjusted pivotal $T_{p}(\hat{\lambda}) / \sqrt{1+c^{2}(\hat{\theta})}$ has approximately the same asymptotic distribution as $T_{p}(\lambda)$ when $n$ is large. However, as discussed in Section 2.1, $\operatorname{Var}\left[T_{p}(\lambda)\right]$ can be far from 1 when $n$ is not large enough, especially when the extreme percentiles are considered. Thus, for small to moderate $n$, the correction factor should be $\sqrt{1+c^{2}(\hat{\theta}) / \sigma_{T}^{2}}$, where $\sigma_{T}^{2}$ is the variance of $T_{p}(\lambda)$ given in (4). Further, as $E\left[T_{p}(\hat{\lambda})\right] \neq 0$ for finite $n$, a change in variance causes a change in the mean, which should then be recovered afterwards. Thus, the adjusted pivotal quantity takes the form

$$
T_{p}^{*}(\hat{\lambda})=\frac{T_{p}(\hat{\lambda})-C_{m}(\hat{\theta})}{C_{s}(\hat{\theta})}
$$

where $C_{m}(\hat{\theta})=\mu_{T}\left(1-C_{s}(\hat{\theta})\right), C_{s}(\hat{\theta})=\sqrt{1+c^{2}(\hat{\theta}) / \sigma_{T}^{2}}$, and $\mu_{t}=E\left[T_{p}(\lambda)\right]$ given in (3). Hence, a handy calibration of the percentile limits factoring into the effects of estimating transformation parameter are obtained as

$$
\left\{\left[1+\hat{\lambda} L^{*}(\hat{\lambda})\right]^{1 / \hat{\lambda}},\left[1+\hat{\lambda} U^{*}(\hat{\lambda})\right]^{1 / \hat{\lambda}}\right\}
$$

with

$$
\begin{aligned}
& L^{*}(\hat{\lambda})=\hat{\mu}(\hat{\lambda})-t_{n-1}^{1-\alpha / 2}\left(-\sqrt{n} z_{p}\right) C_{s}(\hat{\theta}) \frac{s(\hat{\lambda})}{\sqrt{n}}+\left(1-C_{s}(\hat{\theta})\right)\left(\sqrt{n} z_{p}+\mu_{T}\right) \frac{s(\hat{\lambda})}{\sqrt{n}} \\
& U^{*}(\hat{\lambda})=\hat{\mu}(\hat{\lambda})-t_{n-1}^{\alpha / 2}\left(-\sqrt{n} z_{p}\right) C_{s}(\hat{\theta}) \frac{s(\hat{\lambda})}{\sqrt{n}}+\left(1-C_{s}(\hat{\theta})\right)\left(\sqrt{n} z_{p}+\mu_{T}\right) \frac{s(\hat{\lambda})}{\sqrt{n}}
\end{aligned}
$$

We shall call the percentile limits specified by (13) the Calibrated Box-Cox Percentile Limits. When $\hat{\lambda}=0$, they become $\left\{\exp \left[L^{*}(0)\right], \exp \left[U^{*}(0)\right]\right\}$.

\section{Small sample behavior of the PLs}

We have performed Monte Carlo experiments for the following purposes: i) to check the finite sample performance of the calibrated Box-Cox PL and its robustness against the distributional specifications, ii) to see the effect of estimating transformation and the necessity of calibration, and iii) to compare the performance of the calibrated Box-Cox PL with the PL designed specifically for a given distribution, and the distribution-free PL.

The simulation process is described as follows. For each random sample of size $n$ generated from a specified distribution, various percentile limits are calculated. For each 
type of PLs, check if it contains the true percentile and record the length of the interval (upper limit - lower limit). Repeat this process 10,000 times. For each type of PLs, for example the calibrated Box-Cox PL, the proportion of the PLs among the 10,000 generated that cover the true percentile gives a Monte Carlo estimate of the true coverage probability of the calibrated Box-Cox PL. Similarly, the average length of these 10,000 PLs gives a Monte Carlo estimate of the expected length of the PL in question.

Four models, the lognormal, Weibull, gamma and the inverse Gaussian, are used in our simulation studies. Note that an exact PL exists for the lognormal (Hanh and Meeker, 1991, p.56) and an approximate PL exists for the Weibull (Nelson, 1982, p.232). The PLs for gamma and inverse Gaussian are either too complicated or not available, but they are included in order to assess the robustness of each of the four PLs against the data distribution. A distribution-free PL is also available (Hahn and Meeker, 1991, p.82), which usually requires a large sample to yield a reasonable performance, especially at the extreme percentiles. For each chosen distribution, four parameter configurations are used to generate a range of population skewness from small to large, thereby capturing the effect of the skewness on the performance of the Box-Cox type PLs. In addition, four sample sizes $(10,20,30$ and 50) are used; and thirteen percentile points $(1 \%, 5 \%, 10 \%, \cdots, 90 \%, 95 \%$, and $99 \%$ ) are considered. All simulations are performed using Fortran 90 on a mainframe at the National University of Singapore.

Selected simulation results are presented in tables, for easy comparisons of the five PLs (calibrated Box-Cox PL, Box-Cox PL, lognormal PL, Weibull PL and distribution-free $\mathrm{PL}$ ) in terms of both the coverage probability and the average length. Detailed simulation results on coverage probabilities of the PLs (except the distribution-free PL) are presented in plots. The distribution-free PL is excluded from the plots as the method breaks down for small and large percentiles when sample sizes are not large enough.

The Lognormal Model. ${ }^{4}$ The lognormal distribution is of particular interest as in this case an exact normality is achievable by transformation and hence pure effects of

\footnotetext{
${ }^{4} \mathrm{~A}$ random variable $Y$ is said to follow a lognormal distribution, denoted by $L N(\mu, \sigma)$, if $\log Y \sim N\left(\mu, \sigma^{2}\right)$. The parameter $\sigma$ controls the skewness of the distribution and as $\sigma$ increases, the population skewness increases quickly.
} 
estimating transformation as well as analytical calibration can be seen. The simulation results are summarized in Table 1 and Figures 1a-1c.

\section{Table 1 and Figure 1 here}

From Table 1, we see that the calibrated Box-Cox PL performs excellently, with the coverage probabilities and the average lengths very close to those of lognormal PL. The effect of calibration is not negligible at the 50th percentile, but negligible at the 20th and 80th percentiles. However we note from Figure 1 that the effect of calibration is very significant at extreme percentiles, including the 1st, 5th, 95th and 99th, respectively. These empirical findings are consistent with the theory (see the discussions following Corollary 1). In general, we find that an adjustment to the Box-Cox PL should be given. The population skewness significantly affects the length of percentile interval, but does not play a significant role when coverage probability is concerned. More detailed observations from the plots are in order. As sample size increases, the coverage probabilities of the calibrated Box-Cox PL move closer to the nominal levels. In addition, the larger the $n$, the more significant is the effect of calibration. Again, these features are consistent with the theoretical results developed in Section 3. Moreover, the Weibull PL, which is a wrong PL against the chosen lognormal, performs rather poorly with its coverage probability varying wildly. The distribution free PL is rather conservative and can be much longer than the lognormal PL and calibrated Box-Cox PL as seen from Table 1.

The Weibull Model. ${ }^{5}$ The Weibull model is one of the most popular models used in duration and event-time analysis. A Weibull random variable is transformable to near (but not exact) normality by raising it to a power of $0.2654 \beta$ (Hernandze and Johnson, 1981; Yang 1999, 2001). The simulation results are summarized in Table 2 and Figures 2a-2c.

\section{Table 2 and Figure 2 here}

From Table 2, we see that the calibrated Box-Cox PL even outperforms the PL specifically designed for the Weibull distribution, for small to moderate sample sizes and

\footnotetext{
${ }^{5}$ The Weibull distribution, denoted by $W B(\nu, \beta)$, has a cumulative distribution function of the form: $1-\exp \left[-(y / \nu)^{\beta}\right]$, where $\nu$ is the scale parameter and $\beta$ is the shape parameter. The skewness of the population is completely controlled by $\beta$, and the smaller the $\beta$, the more skewed is the distribution.
} 
for percentile points ranging from $10 \%$ to $90 \%$. This is particularly true for the $99 \%$ PLs. In fact, the Weibull PL can be rather liberal when the sample size is small. When the wrong lognormal PL is used for data generated from the Weibull population, its performance is rather unstable. The distribution-free PL is again conservative and can be much longer than the calibrated Box-Cox PL and the Weibull PL. It should be pointed out that a Weibull random variable can not be transformed to exact normality by power transformation. Hence, it is not necessarily true that the calibrated Box-Cox PL has the correct asymptotic coverage. However, our simulation results show that such a non-normality effect is small except at the extreme percentile points. Similar to the case of lognormal distribution, population skewness does not seem to play an important role except in changing the length of the confidence intervals.

The Gamma Model. ${ }^{6}$ The purpose of including the gamma and the following inverse Gaussian model in the simulation is to understand the performance of each of the PLs, particularly when none of the PLs is the right one. Note that a gamma random variable is also transformable to near normality by a power transformation (Hernandze and Johnson, 1981), where the power depends only on the shape parameter and it approaches to $1 / 3$ as the shape parameter goes to $\infty$. The simulation results are summarized in Table 3 and Figures 3a-3c.

\section{Table 3 and Figure 3 here}

As can be observed from Table 3 and Figure 3 only the calibrated Box-Cox PL generally performs in an acceptable way. All other PLs can perform rather poorly at certain situations, with coverage probabilities fluctuating wildly with the change of the population skewness and the percentile point. The population skewness affects the PL behavior in both the coverage probability and the average length. From Figure 3, it seems that the calibrated Box-Cox PL performs better when the population is not so skewed compared with the case when the population is very skewed. An increase in sample size generally improves the

\footnotetext{
${ }^{6}$ The pdf of the gamma distribution is of the form: $\frac{1}{\Gamma(\beta) \nu^{\beta}} y^{\beta-1} \exp (-y / \nu)$, where as in the case of Weibull distribution, $\nu$ is the scale parameter, $\beta$ is the shape parameter, and the value of $\beta$ completely controls the population skewness.
} 
coverage of the calibrated Box-Cox PL, except at the extreme percentile points and with a highly skewed population. These reflect the nonormality effect as the more skewed the gamma population is, the less transformable it is to normality.

The inverse Gaussian Model. ${ }^{7}$ Once again, the inverse Gaussian distribution is transformable to near normality by a simple power transformation with its power depending only on the ratio of the two parameters. The simulation results for the inverse Gaussian model are summarized in Table 4 and Figures $4 a-4 c$.

\section{Table 4 and Figure 4 here}

Similar to the case of gamma distribution, the simulation results indicate that only the calibrated Box-Cox PL performs acceptably in general. All other intervals can perform poorly at certain situations. Also, similar to the gamma case, increasing the sample size generally improves the coverage of the calibrated Box-Cox PL, except the $90 \%$ and $99 \%$ confidence limits for the extreme percentiles of a highly skewed population. These again reflect the effect of non-normality.

Based on the combined analysis of all the simulation results given above, we may conclude that the calibrated Box-Cox PL is very robust with respect to the distributional assumptions. Our findings have bearing on practical applications as to which of the duration or lifetime model should be used to fit the data.

\section{Empirical illustration}

We now use the well-known strike duration data of Kennan (1985) to illustrate the methods of constructing percentile limits introduced in this paper. The data set is reproduced as follows: 79131426295213093741495211931719287299104114152 1532161561982258531012333481122232732333543434410054921212 2121273842 117. They represent the durations in days of 62 strikes in the period from 1968 to 1976 . It has been subsequently analyzed by many authors including Keifer (1988)

\footnotetext{
${ }^{7}$ The pdf of inverse Gaussian is of the form: $\left(\frac{\beta}{2 \pi y^{3}}\right)^{1 / 2} \exp \left(-\frac{\beta(y-\nu)^{2}}{2 \nu^{2} y}\right)$, where $\nu$ is the location parameter, $\beta$ is the scale parameter, and the population skewness is $3(\nu / \beta)^{1 / 2}$.
} 
and Greene (2000), using models such as exponential, lognormal, log logistic and Weibull. The data is positively skewed. We fit the Box-Cox model to the data and obtain the MLE of $\lambda$ being $\hat{\lambda}=0.1668$. The goodness of fit tests indicate that the Box-Cox model fits the data better than other duration models.

The four types of percentile limits are calculated at $p=0.05, .1, .2, .3, .4, .5, .6, .7$, $.8, .9, .95$, and the resultant lower and upper limits are plotted in Figure 5. The Box-Cox estimates of the percentiles are included in the same plots. For easy comparisons, the plots at each nominal coverage level are done in two parts: one corresponding to $p=.05, .1, .3, .4, .5$ and the other corresponding to $p=.6, .7, .8, .9, .95$. From the plots in Figure 5, we see that fitting different models to the data results in substantial different PLs. We recommend the calibrated Box-Cox PL as most adequate on grounds that it is robust against the distributional assumptions and that it is similar in width to the other PLs.

\section{Figure 5 here}

Besides the unified approach to constructing the PLs, we have experimented with some distribution-free methods. As often encountered, the distribution-free method breaks down at $p=0.05$ and 0.95 even for such a sample of size $n=62$. We thus did not include it in the plots of Figure 5. However, we have calculated a few of the distribution-free PL and compared them with the calibrated Box-Cox PL. The results are summarized in Table 5. Consistently, the calibrated Box-Cox PLs are generally shorter.

Table 5: Selected PLs for strike duration data

\begin{tabular}{|c|c|c|c|c|c|c|c|c|c|c|c|c|}
\hline \multirow[b]{2}{*}{$p$} & \multicolumn{6}{|c|}{ Calibrated Box-Cox PL } & \multicolumn{6}{|c|}{ Distribution-free PL } \\
\hline & 90 & $\mathrm{PL}$ & $95^{c}$ & $\mathrm{PL}$ & 99 & $\mathrm{PL}$ & 90 & $\mathrm{PL}$ & 95 & $\mathrm{PL}$ & 99 & $\mathrm{PL}$ \\
\hline .1 & 2.3 & 6.1 & 2.0 & 6.6 & 1.5 & 7.5 & 2 & 5 & 2 & 5 & 1 & 8 \\
\hline .2 & 5.3 & 11.4 & 4.8 & 12.1 & 3.9 & 13.5 & 3 & 12 & 3 & 12 & 3 & 14 \\
\hline .8 & 52.1 & 87.8 & 49.8 & 92.9 & 45.7 & 104.4 & 49 & 114 & 44 & 117 & 43 & 119 \\
\hline .9 & 79.7 & 143.1 & 75.9 & 152.8 & 69.2 & 174.9 & 98 & 152 & 98 & 153 & 72 & 216 \\
\hline
\end{tabular}

\section{Concluding remarks}

In this paper, we have proposed a unified approach to analytically calibrating the Box-Cox type "plug-in" percentile limits for a percentile of a future duration or event- 
time. Percentile limits based on the correct variance of the Box-Cox pivotal quantity are duly constructed. We also examine the small-sample properties of the proposed Box-Cox type percentile limits using Monte Carlo simulations. We find that the Box-Cox PLs are quite robust against the distributional assumptions for duration and event-time models and that it does not require a large sample for the PL to function well. Based on Kennan's strike duration data, we illustrate that the Box-Cox PLs are superior to other PLs under various distributional assumptions. Our findings may help to shed light on a topic for future research where exogenous variables affect the duration or event-time.

There are two interesting issues remain to be resolved: one is to compare our calibration approach with that based on inverting the likelihood ratio test in constructing confidence intervals for $y_{p}$ (see, e.g., Hanh and Meeker, 1991, ch. 12), and the other is to compare the proposed calibration methodology with some extreme quantile estimators proposed in the extreme value literature when the main interest is in extrapolation (see, e.g., Embrecgts, et al., 1997, ch. 6). ${ }^{8}$ However, both issues are quite involved either computationally or theoretically, we decide to deal with them in a separate paper.

\section{Acknowledgements}

The authors would like to thank a referee for the helpful comments. Thanks are also due to the workshop participants at The 2001 Joint Meetings of the Statistical Society of Canada, Western North America Region of the International Biometric Society, and the Institute of Mathematical Statistics.

\section{Appendix}

Proof of Lemma 1. Let $\theta=(\beta, \sigma)^{\prime}, U_{1}(\theta, \lambda)$ and $U_{2}(\theta, \lambda)$ be the score functions that correspond to the estimation of $\theta$ and $\lambda$, respectively. For example, when $\lambda$ is known, then $U_{1}(\theta, \lambda)=0$ defines $\hat{\theta}(\lambda)=[\hat{\beta}(\lambda), \hat{\sigma}(\lambda)]^{\prime}$. Let $I$ be the expected Fisher information matrix, partitioned according to $\theta$ and $\lambda$, with its elements denoted by $I_{\theta \theta}, I_{\theta \lambda}, I_{\lambda \theta}$, and

\footnotetext{
${ }^{8}$ The authors are grateful to an anonymous referee for raising these two issues.
} 
$I_{\lambda \lambda}$. It is easy to see that

$$
\begin{aligned}
\sqrt{n}(\hat{\theta}(\lambda)-\theta) & =-\frac{1}{\sqrt{n}} I_{\theta \theta}^{-1} U_{1}(\theta, \lambda)+o_{p}(1) \\
\sqrt{n}(\hat{\lambda}-\lambda) & =-\frac{1}{\sqrt{n}} I^{\lambda \lambda} U_{2}(\theta, \lambda)+\frac{1}{\sqrt{n}} I^{\lambda \lambda} I_{\theta \lambda} I_{\theta \theta}^{-1} U_{1}(\theta, \lambda)+o_{p}(1)
\end{aligned}
$$

where $I^{\lambda \lambda}$ is the lower-right corner element in $I^{-1}$. From these two expansions, we have

$$
\operatorname{Cov}[\sqrt{n}(\hat{\theta}(\lambda)-\theta), \sqrt{n}(\hat{\lambda}-\lambda)]=o_{p}(1)
$$

hence $\sqrt{n}(\hat{\theta}(\lambda)-\theta)$ is asymptotically independent of $\sqrt{n}(\hat{\lambda}-\lambda)$. As $T_{p}(\lambda)$ depends on $\mathbf{Y}$ only through $\hat{\theta}(\lambda)$, it follows that $T_{p}(\lambda)$ is asymptotically independent of $\sqrt{n}(\hat{\lambda}-\lambda)$.

Proof of Theorem 1. It can be shown that

$$
\bar{h}_{\lambda}(\mathbf{Y}, \lambda)=E\left[h_{\lambda}\left(Y_{0}, \lambda\right)\right]+O_{p}\left(n^{-1 / 2}\right)
$$

By Taylor expansions, we have

$$
\begin{aligned}
\hat{\mu}(\hat{\lambda})+s(\hat{\lambda}) z_{p} & =\hat{\mu}(\lambda)+s(\lambda) z_{p}+(\hat{\lambda}-\lambda)\left[\bar{h}_{\lambda}(\mathbf{Y}, \lambda)+s_{\lambda}(\lambda) z_{p}\right]+O_{p}\left(n^{-1}\right) \\
& =\hat{\mu}(\lambda)+s(\lambda) z_{p}+(\hat{\lambda}-\lambda)\left\{E\left[h_{\lambda}\left(Y_{0}, \lambda\right)\right]+E\left[s_{\lambda}(\lambda)\right] z_{p}\right\}+O_{p}\left(n^{-1}\right), \\
h\left(y_{p}, \hat{\lambda}\right) & =h\left(y_{p}, \lambda\right)+(\hat{\lambda}-\lambda) h_{\lambda}\left(y_{p}, \lambda\right)+O_{p}\left(n^{-1}\right) .
\end{aligned}
$$

It follows that

$$
\begin{aligned}
\hat{\mu}(\hat{\lambda})+ & s(\hat{\lambda}) z_{p}-h\left(y_{p}, \hat{\lambda}\right)=\hat{\mu}(\lambda)+s(\lambda) z_{p}-h\left(y_{p}, \lambda\right) \\
& +(\hat{\lambda}-\lambda)\left[E h_{\lambda}\left(Y_{0}, \lambda\right)+E s_{\lambda}(\lambda) z_{p}-h_{\lambda}\left(y_{p}, \lambda\right)\right]+O_{p}\left(n^{-1}\right) .
\end{aligned}
$$

In addition,

$$
\frac{1}{s(\hat{\lambda})}=\frac{1}{s(\lambda)}+O_{p}\left(n^{-1 / 2}\right)=\frac{1}{\sigma}+O_{p}\left(n^{-1 / 2}\right) .
$$

Combining the above leads to (9) and then (10). Finally, (11) follows from Lemma 1.

Proof of Corollary 1. First, the derivative of $h(., \lambda)$ with respect to $\lambda$ is,

$$
h_{\lambda}\left(Y_{0}, \lambda\right)=\frac{d}{d \lambda} h\left(Y_{0}, \lambda\right)=\frac{1}{\lambda}\left[Y_{0}^{\lambda} \log Y_{0}-h\left(Y_{0}, \lambda\right)\right],
$$

which converges to $\frac{1}{2} \log ^{2} Y_{0}$ as $\lambda$ converges to zero. 
Second, when $\lambda \neq 0, \lambda \log Y_{0}=\log \left(1+\lambda h\left(Y_{0}, \lambda\right)\right)$. A Taylor expansion leads to

$$
\lambda \log Y_{0} \approx \log (1+\lambda \mu)+\theta e_{0}-\frac{1}{2} \theta^{2} e_{0}^{2},
$$

where $\theta=\lambda \sigma /(1+\lambda \mu)$, and $e_{0}=\left[h\left(Y_{0}, \lambda\right)-\mu\right] / \sigma$. After some algebra, it follows that

$$
h_{\lambda}\left(Y_{0}, \lambda\right) \approx \frac{1}{\lambda^{2}} \phi \log \phi+\frac{\sigma}{2 \lambda} \theta e_{0}^{2}+\frac{\sigma}{\lambda} e_{0} \log \phi-\frac{\sigma}{2 \lambda} \theta^{2} e_{0}^{3}-\frac{\mu}{\lambda} .
$$

and

$$
h_{\lambda}\left(y_{p}, \lambda\right) \approx \frac{1}{\lambda^{2}} \phi \log \phi+\frac{\sigma}{2 \lambda} \theta z_{p}^{2}+\frac{\sigma}{\lambda}(\log \phi) z_{p}-\frac{\sigma}{2 \lambda} \theta^{2} z_{p}^{3}-\frac{\mu}{\lambda} .
$$

Similarly, we differentiate $s(\lambda)$ with respect to $\lambda$ to get

$$
\begin{aligned}
s_{\lambda}(\lambda) & =\frac{d}{d \lambda}\left\{\frac{1}{n-1} \sum_{i=1}^{n}\left[h\left(Y_{i}, \lambda\right)-\bar{h}(Y, \lambda)\right]^{2}\right\}^{1 / 2} \\
& =\frac{1}{(n-1) s(\lambda)}\left\{\sum_{i=1}^{n}\left[h\left(Y_{i}, \lambda\right)-\bar{h}(Y, \lambda)\right]\left[h_{\lambda}\left(Y_{i}, \lambda\right)-\bar{h}_{\lambda}(Y, \lambda)\right]\right\} \\
& =\frac{1}{(n-1) \sigma}\left\{\sum_{i=1}^{n}\left[h\left(Y_{i}, \lambda\right)-\bar{h}(Y, \lambda)\right] h_{\lambda}\left(Y_{i}, \lambda\right)\right\}+O_{p}\left(n^{-1 / 2}\right) \\
& =\frac{1}{n-1} \sum_{i=1}^{n} e_{i} h_{\lambda}\left(Y_{i}, \lambda\right)+O_{p}\left(n^{-1 / 2}\right) \\
& =E\left[e_{0} h_{\lambda}\left(Y_{0}, \lambda\right)\right]+O_{p}\left(n^{-1 / 2}\right)
\end{aligned}
$$

Since $h\left(Y_{0}, \lambda\right)$ is approximate normal, the first four moments of $e_{0}$ are approximately the same as those of the standard normal random variable. Thus,

$$
E\left[h_{\lambda}\left(Y_{0}, \lambda\right)\right] \approx \frac{1}{\lambda^{2}} \phi \log \phi+\frac{\sigma}{2 \lambda} \theta
$$

and

$$
E\left[e_{0} h_{\lambda}\left(Y_{0}, \lambda\right)\right] \approx \frac{\sigma}{\lambda} \log \phi-\frac{3}{2} \frac{\sigma}{\lambda} \theta^{2}
$$

A little calculation yields

$$
E\left[h_{\lambda}\left(Y_{0}, \lambda\right)\right]+E\left[s_{\lambda}(\lambda)\right] z_{p}-h_{\lambda}\left(y_{p}, \lambda\right) \approx \frac{\sigma \theta}{2 \lambda}\left(1-z_{p}^{2}\right)-\frac{\sigma \theta^{2}}{2 \lambda}\left(3 z_{p}-z_{p}^{3}\right) .
$$

Finally, we use a result from Yang (1999a). That is

$$
\operatorname{Var}[\sqrt{n}(\hat{\lambda}-\lambda)] \approx 2 \lambda^{2} /\left(3 \theta^{2}\right)
$$


Putting the relevant approximations together gives the results as stated in Corollary 1.

\section{References}

1. Baker, M. and Melino, A. (2000). Duration dependence and nonparametric heterogeneity: A Monte Carlo study. Journal of Econometrics, 96, 357-393.

2. Box, G. E. P and Cox, D. R. (1964). An analysis of transformation (with discussion). Journal of the Royal Statistical Society B 26, 211-252.

3. Carroll, R. J. and Ruppert, D. (1981). On prediction and the power transformation family. Biometrika 68, 609-615.

4. Collins, S. (1991). Prediction techniques for Box-Cox regression models. Journal of Business and Economic Statistics 9, 267-277.

5. Embrechts, P., Klüppelberg, C. and Mikosch, T. (1997). Modelling Extremal Events for Insurance and Finance. Berlin: Springer.

6. Engel, R. F. and Russell, J. R. (1998). Autoregressive conditional duration: A new model for irregular spaced transaction data. Econometrica 66, 1127-1162.

7. Greene, W. H. (2000). Econometric Analysis, 4th ed. Prentice-Hall, Inc.

8. Hahn, G. J. and Meeker, W. Q. (1991). Statistical Intervals. A guide for Practitioners. New York: John Wiley \& Sons, Inc.

9. Heckman, J. and Singer, B. (1984a). Econometric duration analysis. Journal of Econometrics 24, 63-132.

10. Heckman, J. and Singer, B. (1984b). A method for minimizing the impact of distributional assumptions in econometric models for duration data. Econometrica 52, 271-320.

11. Hernandze, F. and Johnson, R. A. (1980). The large-sample behavior of transformations to normality. Journal of American Statistical Association 75, 855-861.

12. Kennan, J. (1985). The duration of contract strikes in U.S. manufacturing. Journal of Econometrics 28, 5-28.

13. Kiefer, N. M. (1988). Economic Duration Data and Hazard Functions. Journal of Economic Literature 26, 646-679. 
14. Kiefer, N. M., Lundberg, S. and Neumann, G. R. (1985). How long is a spell of unemployment? Journal of Business and Economic Statistics 3, 118-128.

15. Koop, G. and Ruhm, C. J. (1993). Econometric estimation of proportional hazard models. Journal of Economics and Business 45, 421-430.

16. Lancaster, T. (1972). A stochastic model for the duration of a strike. Journal of the Royal Statistical Society A 135, 2, 257-271.

17. Lancaster, T. (1979). Econometric methods for the duration of unemployment. Econometrica $47,939-956$.

18. Lancaster, T. (1985). Generalised residuals and heterogeneous duration models - with applications to the Weibull model. Journal of Econometrics 28, 113-126.

19. Lancaster, T. (1990). The Econometric Analysis of Transition Data. Cambridge: Cambridge University Press.

20. Nelson, W. (1982). Applied Life Data Analysis. New York: Wiley.

21. Ryu, K. K. (1993). Structural duration analysis of management data. Journal of Econometrics 57, 91-115.

22. Saha, A. and Hilton, L. (1997). Expo-power: a flexible hazard function for duration models. Economics Letters 54, 227-233.

23. Sider, H. (1985). Unemployment duration and incidence: 1968-82. American Economic Review. 75, 461-472.

24. Torelli, N. and Trivellato, U. (1993). Modelling inacuracies in job-search duration data. Journal of Econometrics 59, 187-211.

25. Yang, Z. (1999a). Estimating a transformation and its effect on Box-Cox T-ratio. Test 8, 167-190.

26. Yang, Z. (1999b). Predicting a future lifetime through Box-Cox transformation. Lifetime Data Analysis 5, 265-279.

27. Yang, Z. (2001). Predicting a future median life through a power transformation. Lifetime Data Analysis 7, 307-319.

28. Zhang, M. Y. J., Russell J. R. and Tsay, R. S. (2001). A nonlinear autoregressive conditional duration model with applications to financial transaction data. Journal of Econometrics 104, 179-207. 
Table 1: Selected simulation results for $L N(\mu, \sigma), \mu=1, n=30$

\begin{tabular}{|c|c|c|c|c|c|c|c|c|c|c|c|}
\hline \multirow[b]{2}{*}{$1-\alpha$} & \multirow[b]{2}{*}{$\sigma$} & \multicolumn{4}{|c|}{ Coverage Probability } & \multicolumn{6}{|c|}{ Average Length } \\
\hline & & $\mathrm{CBC}$ & $\mathrm{BC}$ & LN & WB & $\mathrm{DF}$ & $\mathrm{CBC}$ & & IN & WB & $\mathrm{DF}$ \\
\hline \multirow{5}{*}{.90} & & & & & & & & & & & \\
\hline & 0.5 & .8945 & .8679 & .8966 & .7151 & .9003 & 0.88 & 0.82 & 0.84 & 0.81 & 1.05 \\
\hline & 1.0 & .89 & .8697 & .9027 & .7245 & .8994 & 1.81 & 1.68 & 1.72 & 1.80 & 2.17 \\
\hline & 1. & .89 & .8672 & .9010 & .7116 & .9007 & 2. & 2.64 & 2.70 & 3.06 & 3.49 \\
\hline & 2.0 & .8918 & .8640 & .9018 & .7155 & .8975 & 4.05 & 3.74 & 3.81 & 4.66 & 5.10 \\
\hline \multirow[t]{4}{*}{.95} & 0.5 & .94 & .9291 & .9476 & .8054 & .9547 & 1. & 0.99 & 1.02 & .97 & 1.31 \\
\hline & 1.0 & .95 & .9325 & .9519 & .8094 & .9580 & 2. & 2.05 & 2.10 & 2.17 & 2.74 \\
\hline & 1.5 & .94 & .9328 & .9510 & .8023 & .9581 & 3.52 & 3.25 & 3.33 & 3.71 & 4.47 \\
\hline & 2.0 & .94 & .9281 & .9490 & .7994 & & $5 .($ & 4.63 & 4.73 & 5.69 & 6.65 \\
\hline \multirow[t]{5}{*}{.99} & 0.5 & .99 & .9846 & .9899 & .9218 & .9936 & 1. & 1.34 & 1.37 & & 1.86 \\
\hline & & & .9847 & .9895 & .9187 & .9951 & 3. & 2.82 & 2.89 & & 4.05 \\
\hline & 1.5 & .99 & .9849 & .9917 & .9225 & .9959 & 4. & 4.54 & 4.66 & 5.00 & 6.89 \\
\hline & 2.0 & .99 & & .9905 & .9211 & .9947 & 7. & 6.71 & 6.87 & 7.88 & 10.9 \\
\hline & & & & & & 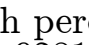 & & & & & \\
\hline \multirow[t]{4}{*}{.90} & 0.5 & .89 & .8 & .8976 & .9208 & .92 & 0.64 & 0.64 & 0.63 & 0.88 & 0.93 \\
\hline & & & & .9045 & .9186 & & & & & & \\
\hline & & & & .9025 & & & & & 0. & & 4 \\
\hline & 2. & .89 & .8900 & .9011 & .9167 & .92 & & 80 & 0.78 & 6 & 1.05 \\
\hline \multirow{4}{*}{.95} & 0. & .9 & .9431 & .9522 & .9559 & & 0. & .76 & 0.76 & & 1.02 \\
\hline & 1. & .94 & .9387 & .9474 & .9590 & & 1. & 1.01 & 1.00 & & 3 \\
\hline & & & .94 & .9511 & .9595 & & 1. & & 1.02 & & $=$ \\
\hline & & & & & & & & & & & \\
\hline \multirow[t]{5}{*}{.99} & 0. & & .98 & & .9881 & & 1. & 1 & 1.01 & & 1.45 \\
\hline & 1 & & & & .9900 & & & & & & \\
\hline & 1 & & & & .9894 & & & & 1.36 & & \\
\hline & 2 & & .9 & .9 & .9898 & .99 & 1.32 & 1. & 1.28 & 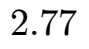 & \\
\hline & & \multicolumn{10}{|c|}{ rcentile } \\
\hline \multirow[t]{4}{*}{90} & 0 . & 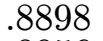 & 88 & .89 & .7782 & & 154 & 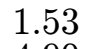 & 1. & & \\
\hline & & & & & .78 & & & & 5. & & \\
\hline & 1 & .88 & & & .78 & & & 12.3 & 12 & & \\
\hline & 2. & & .88 & .89 & .77 & & & 27.6 & 28 & & \\
\hline \multirow[t]{4}{*}{.95} & 0. & .94 & .9412 & .9524 & .8533 & & & & 1.89 & & 2.89 \\
\hline & & & & & & & & & 6.24 & & \\
\hline & 1 & & & & & & & & 15.8 & & .1 \\
\hline & 2 & .94 & & .95 & .85 & & & 36.0 & 37.0 & & \\
\hline \multirow[t]{4}{*}{.99} & 0. & .9 & & .99 & .93 & & & 2.61 & 2.63 & & 4.77 \\
\hline & 1. & .98 & .98 & & .9352 & & 9. & 8.94 & 9.01 & & 20.6 \\
\hline & 1. & & & & & & & 24 & 24.5 & 13.4 & 75.9 \\
\hline & 2.0 & .98 & .9832 & .9906 & .9363 & .99 & 62.4 & 60.8 & 60.9 & 29.6 & 298 \\
\hline
\end{tabular}

Notes: CBC, BC, LN, WB and DF stand, respectively, for calibrated Box-Cox PL, Box-Cox PL, lognormal PL, Weibull PL and distribution-free PL. 
Table 2: Selected simulation results for $W B(\nu, \beta), \nu=1, n=30$

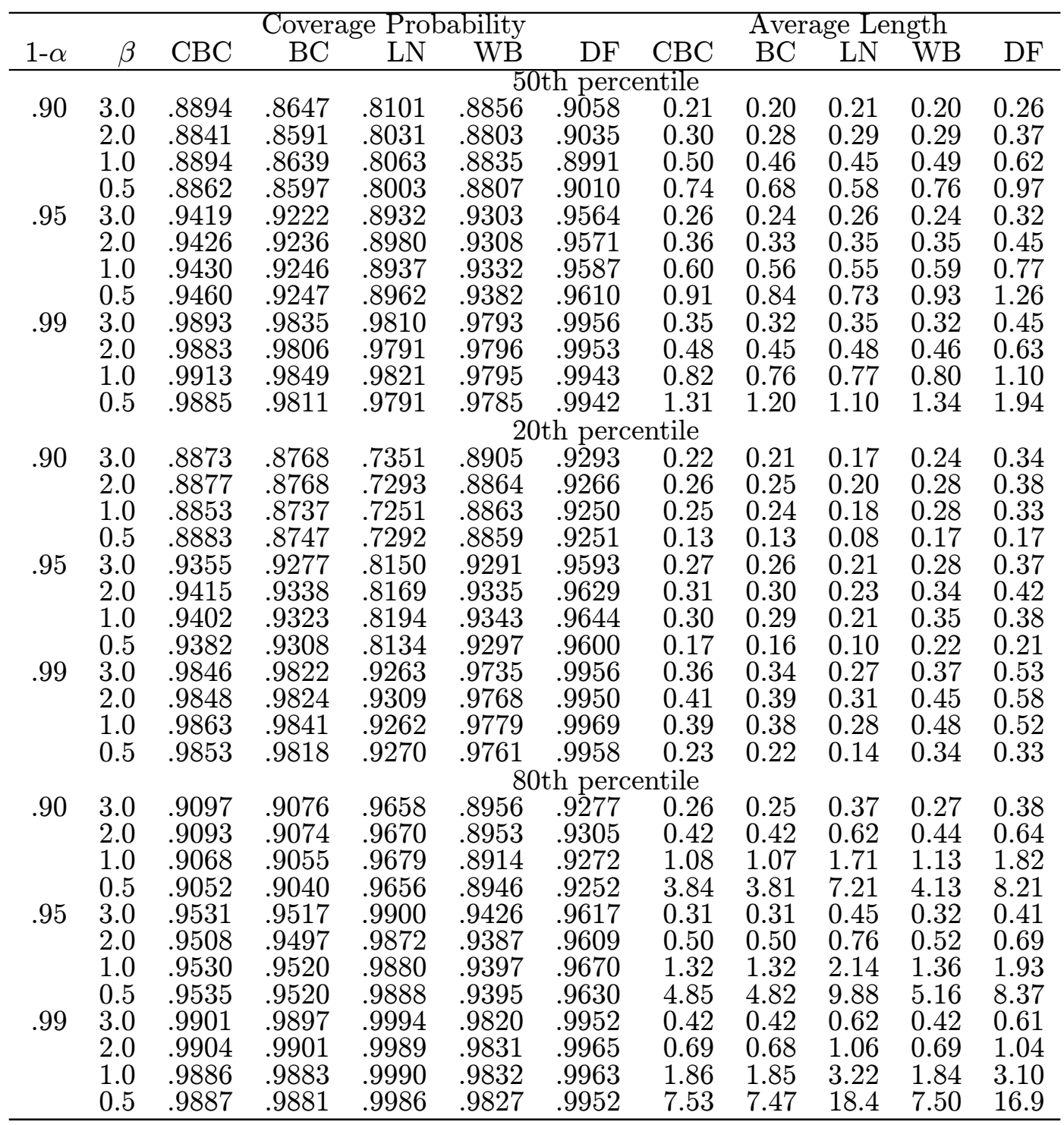

Notes: CBC, BC, LN, WB and DF stand, respectively, for calibrated Box-Cox PL, Box-Cox PL, lognormal PL, Weibull PL and distribution-free PL. 
Table 3: Selected simulation results for $G A(\nu, \beta), \nu=1, n=30$

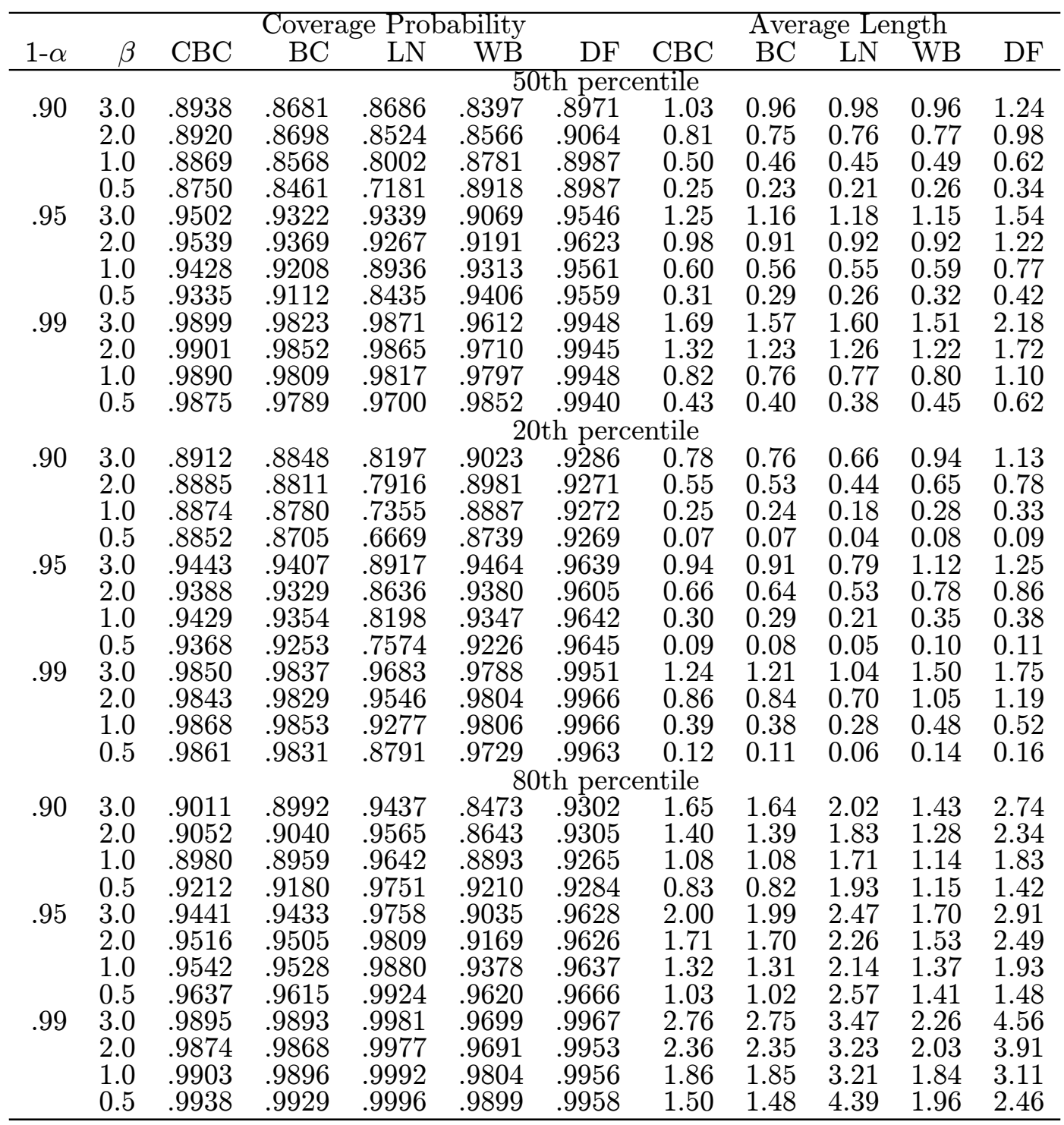

Notes: CBC, BC, LN, WB and DF stand, respectively, for calibrated Box-Cox PL, Box-Cox PL, lognormal PL, Weibull PL and distribution-free PL. 
Table 4: Selected simulation results for $I G(\nu, \beta), \nu=1, n=30$

\begin{tabular}{|c|c|c|c|c|c|c|c|c|c|c|c|}
\hline \multirow[b]{2}{*}{$1-\alpha$} & \multirow[b]{2}{*}{$\beta$} & & \multicolumn{3}{|c|}{ Coverage Probability } & \multirow[b]{2}{*}{ DF } & \multicolumn{5}{|c|}{ Average Length } \\
\hline & & $\mathrm{CBC}$ & $\mathrm{DC}$ & LN & WB & & $\mathrm{CBC}$ & $\mathrm{BC}$ & LN & WB & DF \\
\hline & & & & & & h per & ntile & & & & \\
\hline \multirow{4}{*}{.90} & 9.00 & .8863 & .8613 & .8970 & .7127 & .8961 & 0.20 & 0.19 & 0.19 & 0.18 & 0.24 \\
\hline & 4.00 & .8921 & .8666 & .8992 & .7026 & .9017 & 0.27 & 0.26 & 0.26 & 0.25 & 0.33 \\
\hline & 1.00 & .8856 & .8603 & .9011 & .6578 & .9071 & 0.38 & 0.35 & 0.37 & 0.38 & 0.48 \\
\hline & 0.25 & .8725 & .8421 & .8631 & .5265 & .9028 & 0.32 & 0.30 & 0.34 & 0.37 & 0.42 \\
\hline \multirow[t]{4}{*}{.95} & 9.00 & .9476 & .9294 & .9510 & .8036 & .9554 & 0.24 & 0.22 & 0.23 & 0.21 & 0.30 \\
\hline & 4.00 & .9468 & .9299 & .9527 & .8045 & .9582 & 0.33 & 0.31 & 0.32 & 0.30 & 0.41 \\
\hline & 1.00 & .9388 & .9185 & .9472 & .7530 & .9570 & 0.46 & 0.43 & 0.45 & 0.45 & 0.60 \\
\hline & 0.25 & .9336 & .9132 & .9295 & .6383 & .9592 & 0.40 & 0.37 & 0.41 & 0.45 & 0.55 \\
\hline \multirow[t]{4}{*}{.99} & 9.00 & .9905 & .9826 & .9897 & .9155 & .9947 & 0.33 & 0.30 & 0.31 & 0.28 & 0.42 \\
\hline & 4.00 & .9893 & .9820 & .9885 & .9189 & .9951 & 0.45 & 0.42 & 0.43 & 0.40 & 0.59 \\
\hline & 1.00 & .9868 & .9797 & .9885 & .8961 & .9945 & 0.63 & 0.59 & 0.61 & 0.60 & 0.88 \\
\hline & 0.25 & .9851 & .9753 & .9842 & .8280 & .9942 & 0.56 & 0.51 & 0.58 & 0.60 & 0.86 \\
\hline & & & & & & per & & & & & \\
\hline \multirow{4}{*}{.90} & 9.00 & .8889 & .8865 & 8998 & .9149 & .9248 & 0.17 & 0.17 & 0.17 & 0.23 & 0.25 \\
\hline & 4.00 & .8941 & .8919 & .9059 & .9099 & .9264 & 0.20 & 0.20 & 0.20 & 0.28 & 0.29 \\
\hline & 1.00 & .9001 & .8987 & .9225 & .9079 & .9270 & 0.20 & 0.20 & 0.21 & 0.31 & 0.26 \\
\hline & 0.25 & .9096 & .9081 & .9492 & .9256 & .9309 & 0.11 & 0.11 & 0.13 & 0.20 & 0.13 \\
\hline \multirow[t]{4}{*}{.95} & 9.00 & .9440 & .9428 & .9524 & .9555 & .9668 & 0.20 & 0.20 & 0.20 & 0.27 & 0.27 \\
\hline & 4.00 & .9457 & .9444 & .9533 & .9527 & .9650 & 0.24 & 0.24 & 0.24 & 0.33 & \\
\hline & 1.00 & .9497 & .9489 & .9657 & .9543 & .9654 & 0.24 & 0.24 & 0.25 & 0.37 & 0.30 \\
\hline & & .9516 & .9505 & .9761 & .9669 & .9647 & 0.13 & 0.13 & 0.15 & 0.25 & 0.16 \\
\hline \multirow[t]{5}{*}{.99} & 9.00 & .9880 & .9873 & .9911 & .9892 & .9967 & 0.27 & 0.27 & 0.27 & 0.36 & 0.39 \\
\hline & 400 & .9873 & .9869 & .9926 & .9884 & .9965 & 0.32 & 0.32 & 0.32 & 0.44 & 0.45 \\
\hline & 1.00 & .9859 & .9859 & .9927 & .9885 & .9968 & 0.31 & 0.31 & 0.33 & 0.50 & 0.42 \\
\hline & \multirow{2}{*}{\multicolumn{11}{|c|}{ 80th percentile }} \\
\hline & & & & & & & & & & & \\
\hline \multirow{4}{*}{.90} & 9.00 & .8934 & .8912 & .9035 & .7729 & .9308 & 0.30 & 0.30 & 0.30 & 0.22 & 0.51 \\
\hline & 100 & .8934 & & .8968 & .7754 & .9264 & 0.48 & 0.47 & 0.47 & 0.34 & \\
\hline & 1.00 & .8960 & .8916 & .8945 & .7577 & .9289 & 0.97 & 0.96 & 0.93 & 0.64 & 1.89 \\
\hline & & .8977 & .8910 & .8714 & .7357 & .9323 & 1.45 & 1.40 & 1.30 & 0.86 & \\
\hline \multirow[t]{4}{*}{.95} & 9.00 & .9473 & .9467 & .9559 & .8541 & .9676 & 0.36 & 0.36 & 0.36 & 0.26 & 0.54 \\
\hline & 4.00 & .9455 & .9438 & .9495 & .8457 & .9643 & 0.58 & 0.57 & 0.57 & 0.40 & 0.87 \\
\hline & 1.0 & .9476 & .9459 & .9475 & .8428 & .96 & 1.2 & 1.19 & 1.15 & 0.77 & 2.00 \\
\hline & 0 . & .9420 & .9380 & .9260 & .8068 & .9651 & 1.87 & 1.81 & 1.64 & 1.04 & 3.78 \\
\hline \multirow[t]{4}{*}{.99} & 9.00 & .9839 & .9835 & .9878 & .9311 & .9939 & 0.50 & 0.50 & 0.50 & 0.34 & 0.84 \\
\hline & 4.00 & .9872 & .9868 & .9911 & .9341 & .9952 & 0.81 & 0.80 & 0.79 & 0.53 & 1.41 \\
\hline & 1.0 & .9877 & .9875 & .9886 & .9226 & .9956 & 1.77 & 1.74 & 1.64 & 1.03 & 3.42 \\
\hline & 0.25 & .9862 & .9854 & .9803 & .9014 & .9955 & 3.11 & 2.97 & 2.49 & 1.42 & 7.61 \\
\hline
\end{tabular}

Notes: CBC, BC, LN, WB and DF stand, respectively, for calibrated Box-Cox PL, Box-Cox PL, lognormal PL, Weibull PL and distribution-free PL. 
Figure 1a. Coverage probabilities of the $90 \%$ PLs: Lognormal data
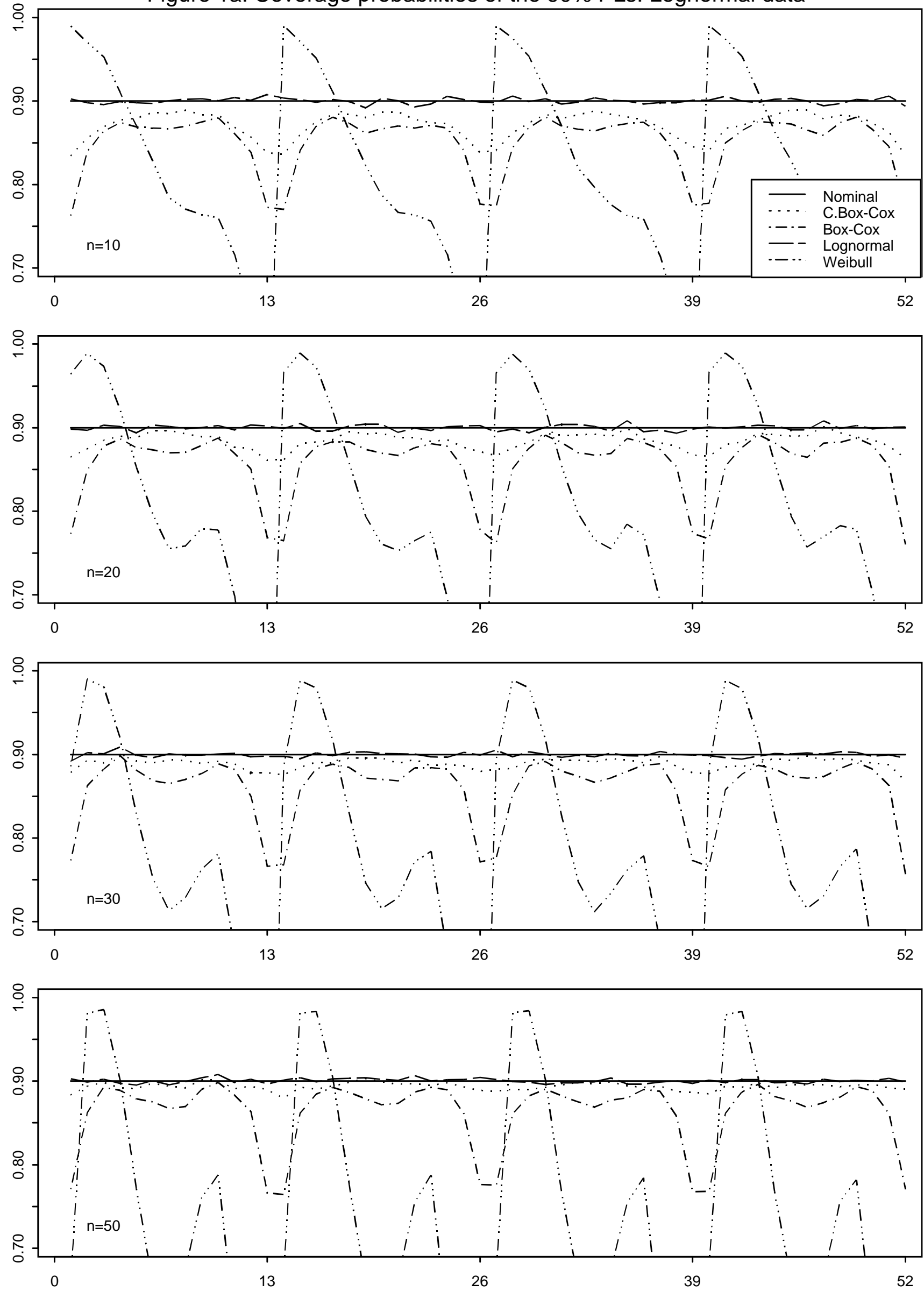

The horizontal index (1-52) corresponds to $\mathrm{p}=.01, .05, .1, .2, .3, .4, .5, .6, .7, .8, .9, .95, .99$, for each of sigma $=.5,1 ., 1.5,2$. 
Figure 1b. Coverage probabilities of the 95\% PLs: Lognormal data
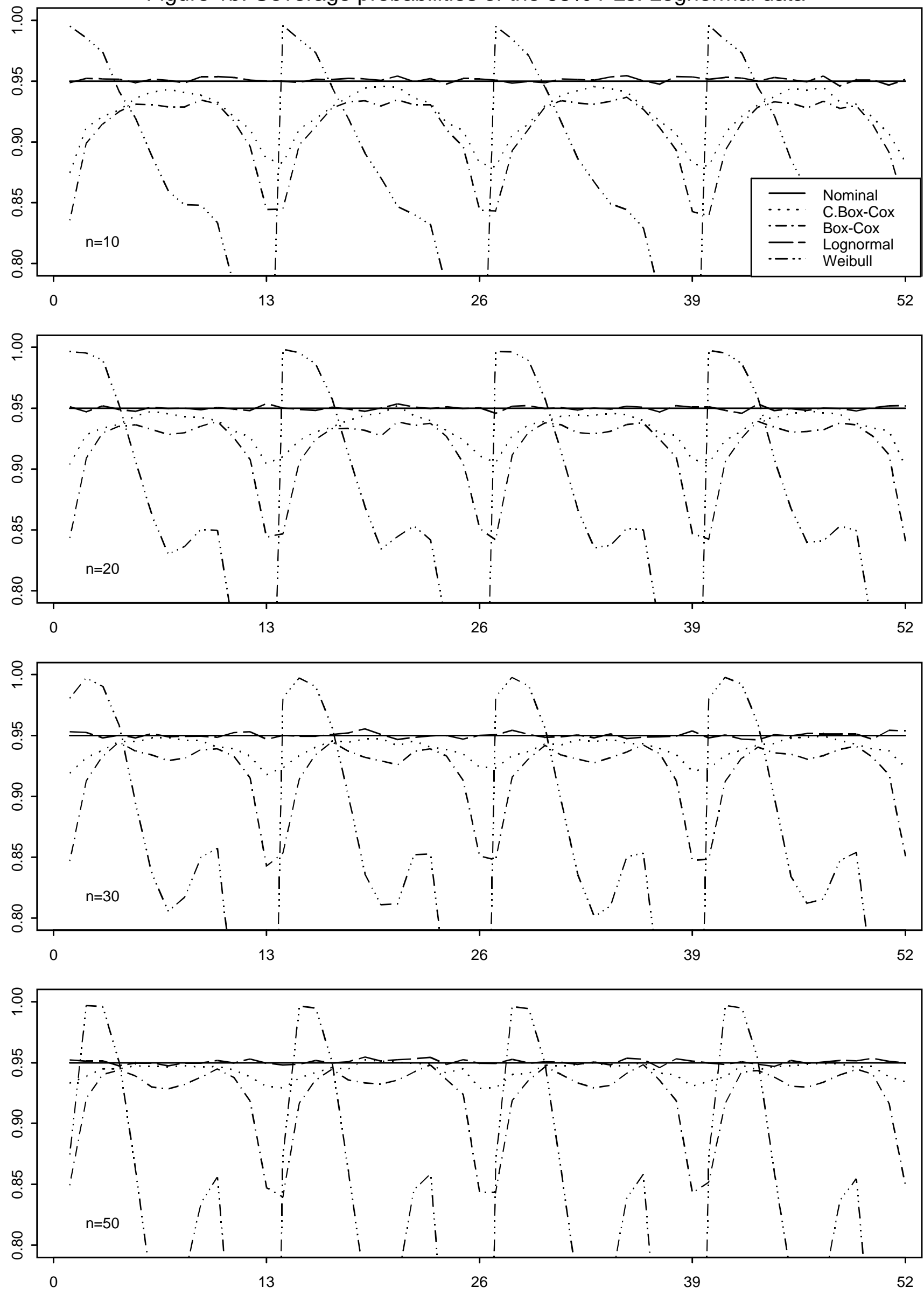

The horizontal index (1-52) corresponds to $\mathrm{p}=.01, .05, .1, .2, .3, .4, .5, .6, .7, .8, .9, .95, .99$, for each of sigma=.5, 1., 1.5, 2 . 
Figure 1c. Coverage probabilities of the 99\% PLs: Lognormal data
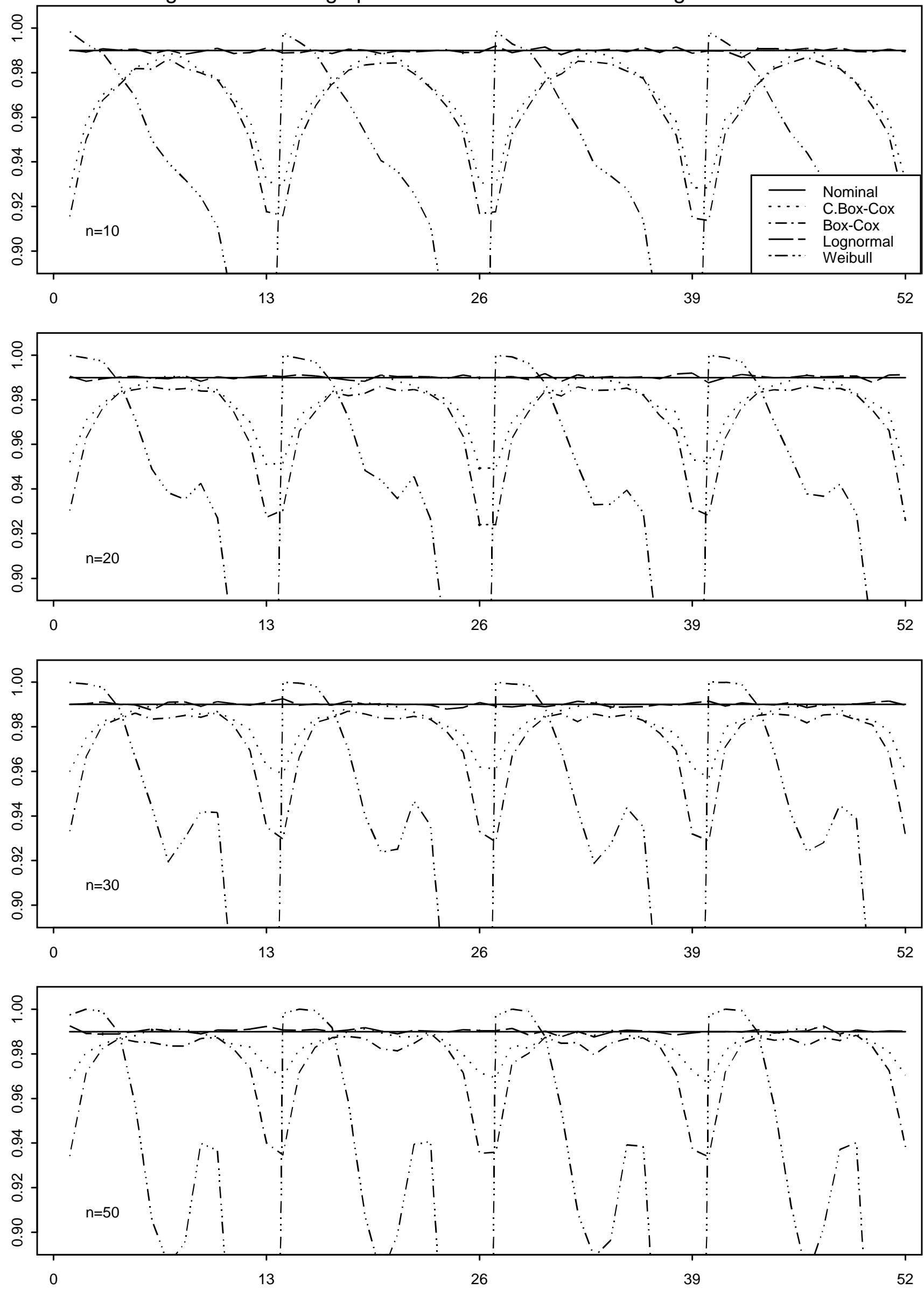

The horizontal index (1-52) corresponds to $\mathrm{p}=.01, .05, .1, .2, .3, .4, .5, .6, .7, .8, .9, .95, .99$, for each of sigma=.5, $1 ., 1.5,2$. 
Figure 2a. Coverage probabilities of the $90 \%$ PLs: Weibull data
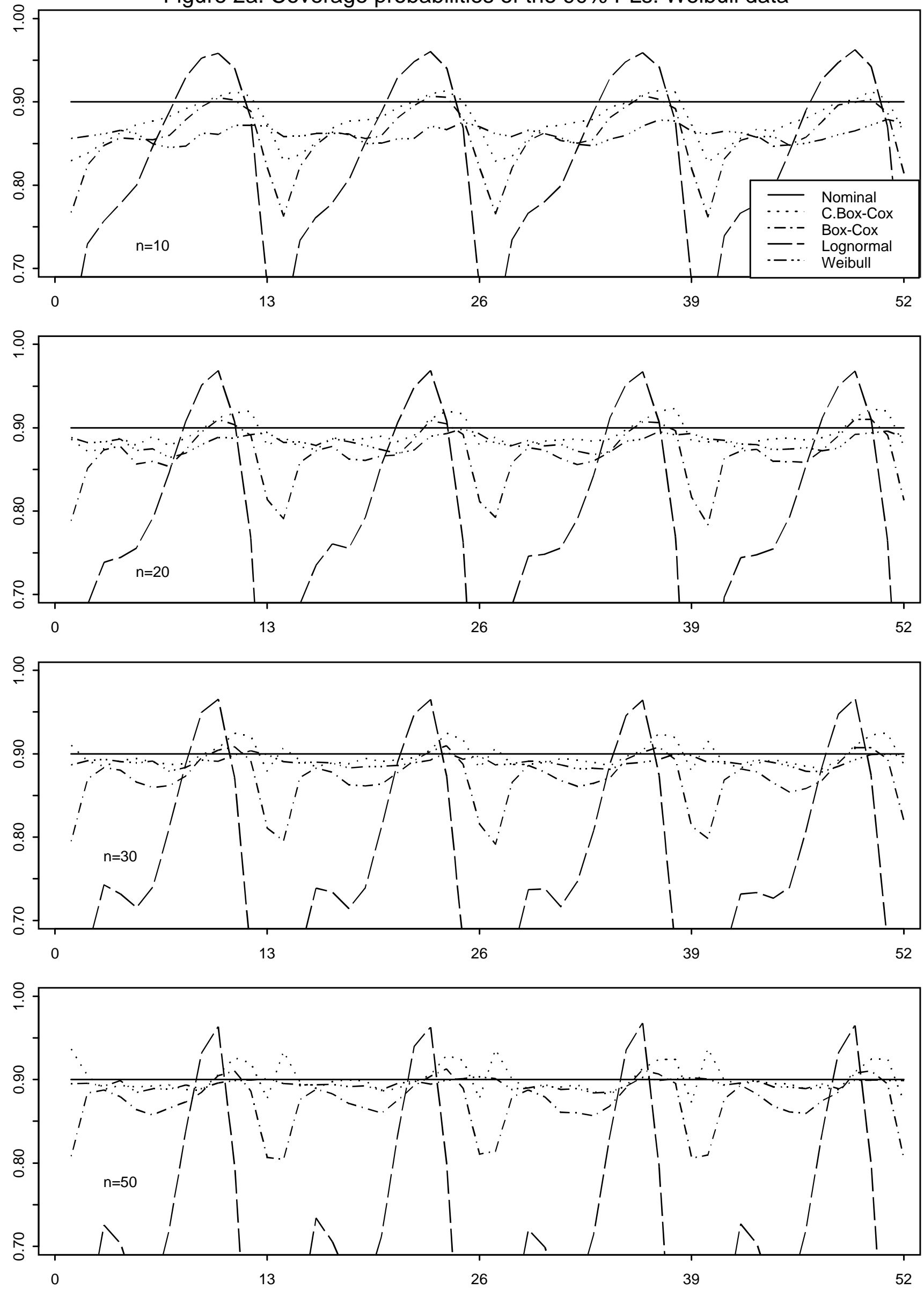

The horizontal index (1-52) corresponds to $\mathrm{p}=.01, .05, .1, .2, .3, .4, .5, .6, .7, .8, .9, .95, .99$, for each of beta $=3,2,1, .5$ 
Figure $2 \mathrm{~b}$. Coverage probabilities of the $95 \%$ PLs: Weibull data
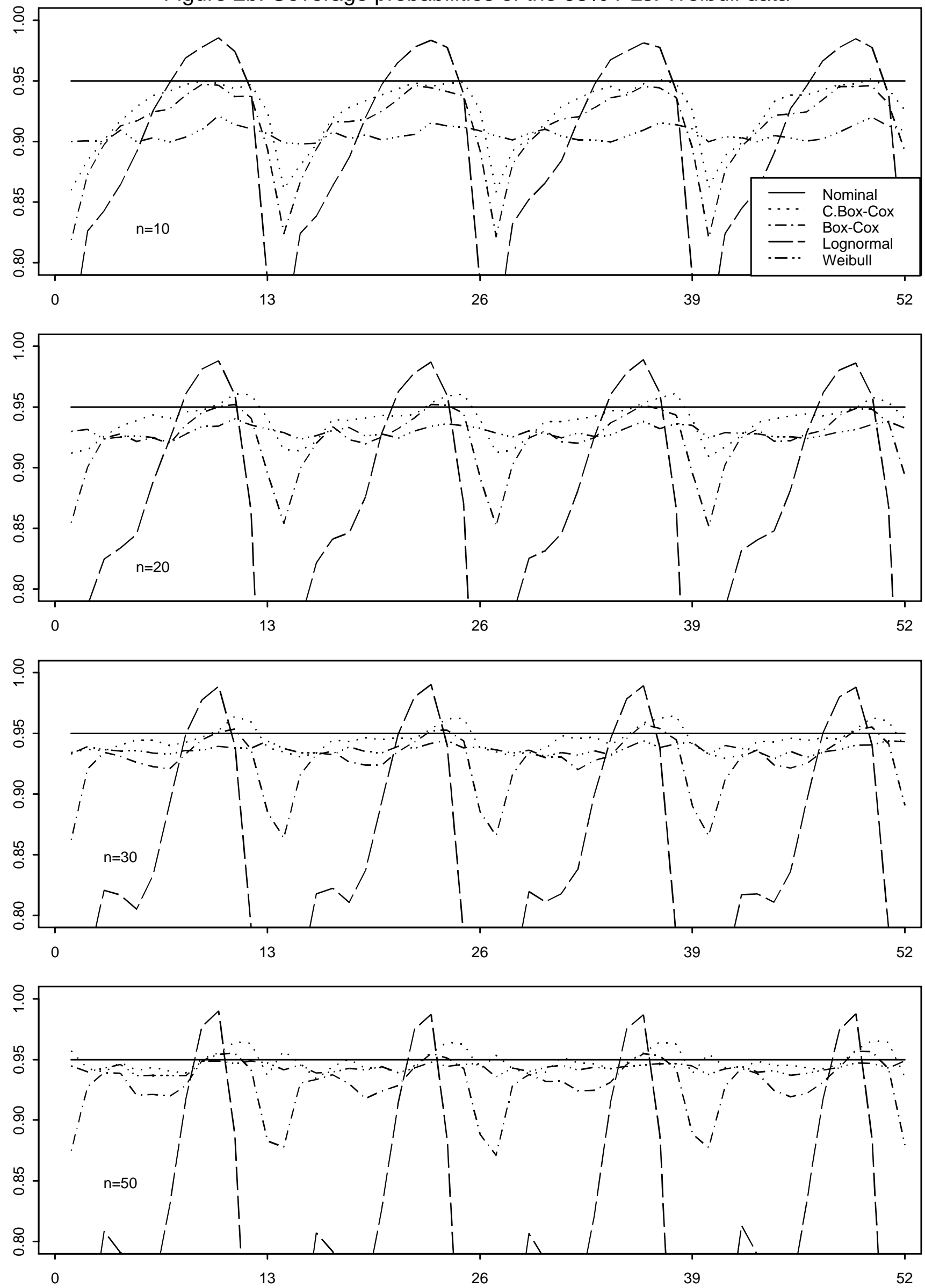

The horizontal index (1-52) corresponds to $\mathrm{p}=.01, .05, .1, .2, .3, .4, .5, .6, .7, .8, .9, .95, .99$, for each of beta=3, $2,1, .5$ 
Figure 2c. Coverage probabilities of the $99 \%$ PLs: Weibull data
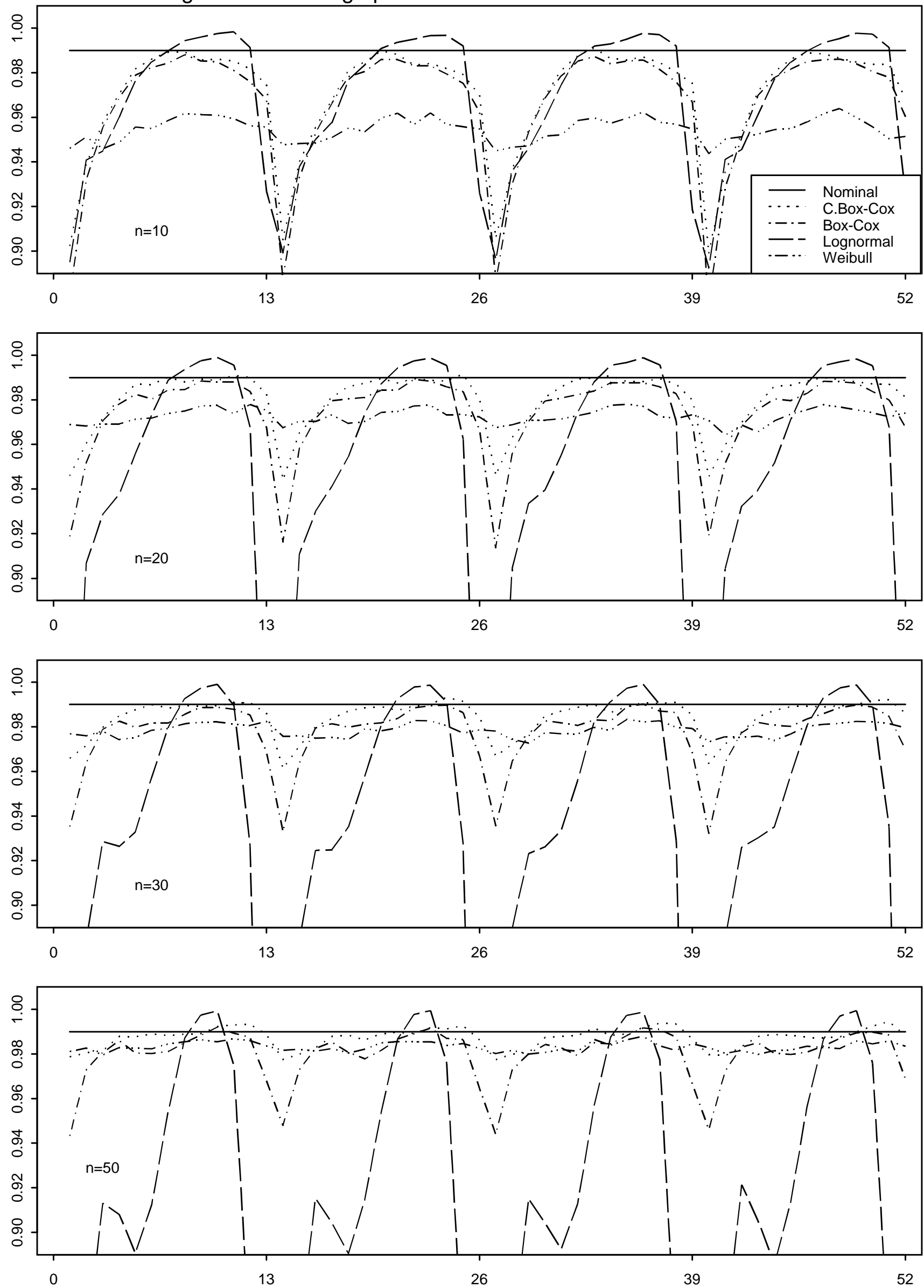

The horizontal index (1-52) corresponds to $\mathrm{p}=.01, .05, .1, .2, .3, .4, .5, .6, .7, .8, .9, .95, .99$, for each of beta=3, $2,1, .5$ 
Figure 3a. Coverage probabilities of the $90 \%$ PLs: Gamma data
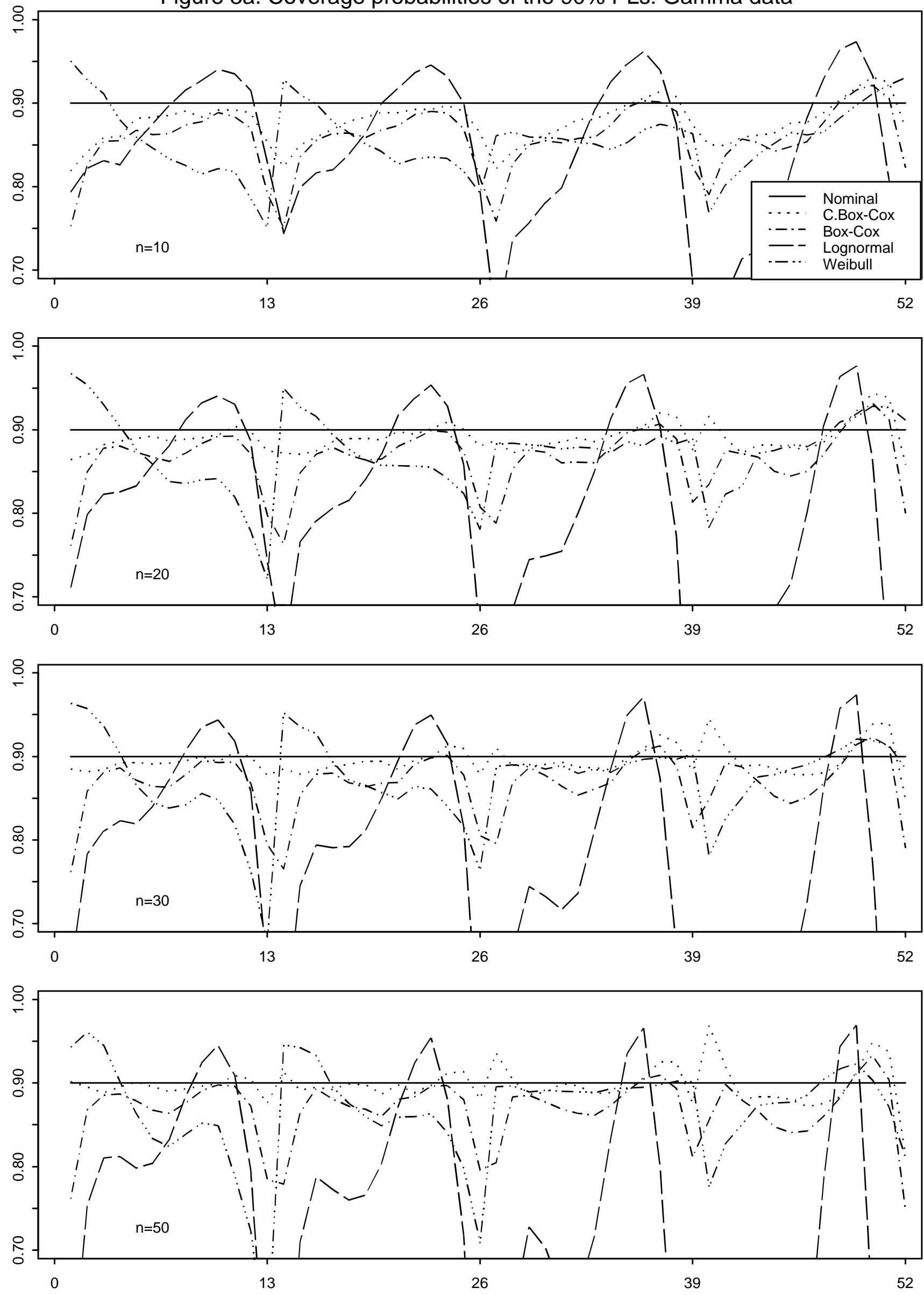

The horizontal index (1-52) corresponds to $\mathrm{p}=.01, .05, .1, .2, .3, .4, .5, .6, .7, .8, .9, .95, .99$, for each of beta $=3,2,1, .5$ 
Figure 3b. Coverage probabilities of the 95\% PLs: Gamma data
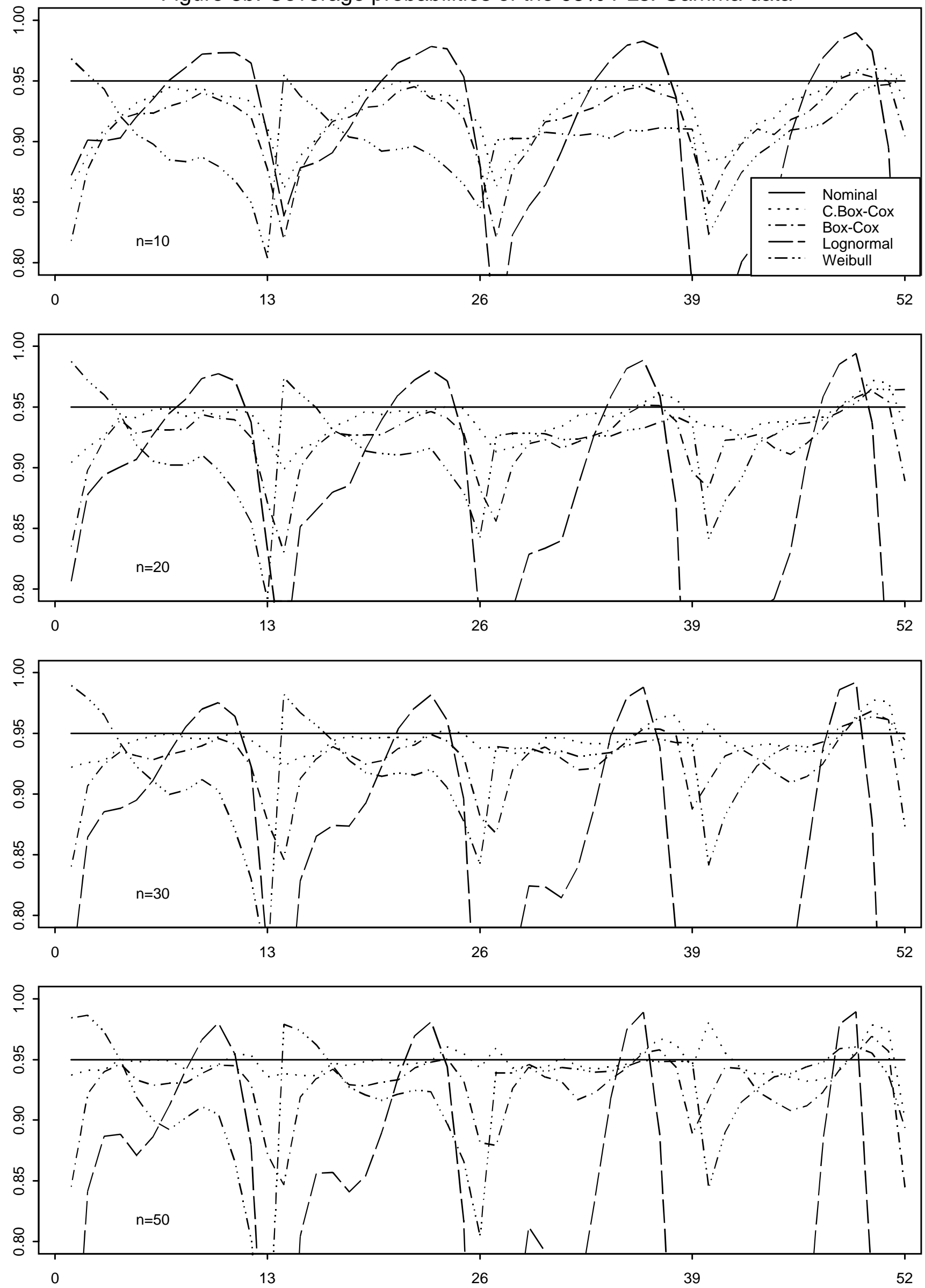

The horizontal index (1-52) corresponds to $\mathrm{p}=.01, .05, .1, .2, .3, .4, .5, .6, .7, .8, .9, .95, .99$, for each of beta=3, $2,1, .5$ 
Figure 3c. Coverage probabilities of the $99 \%$ PLs: Gamma data
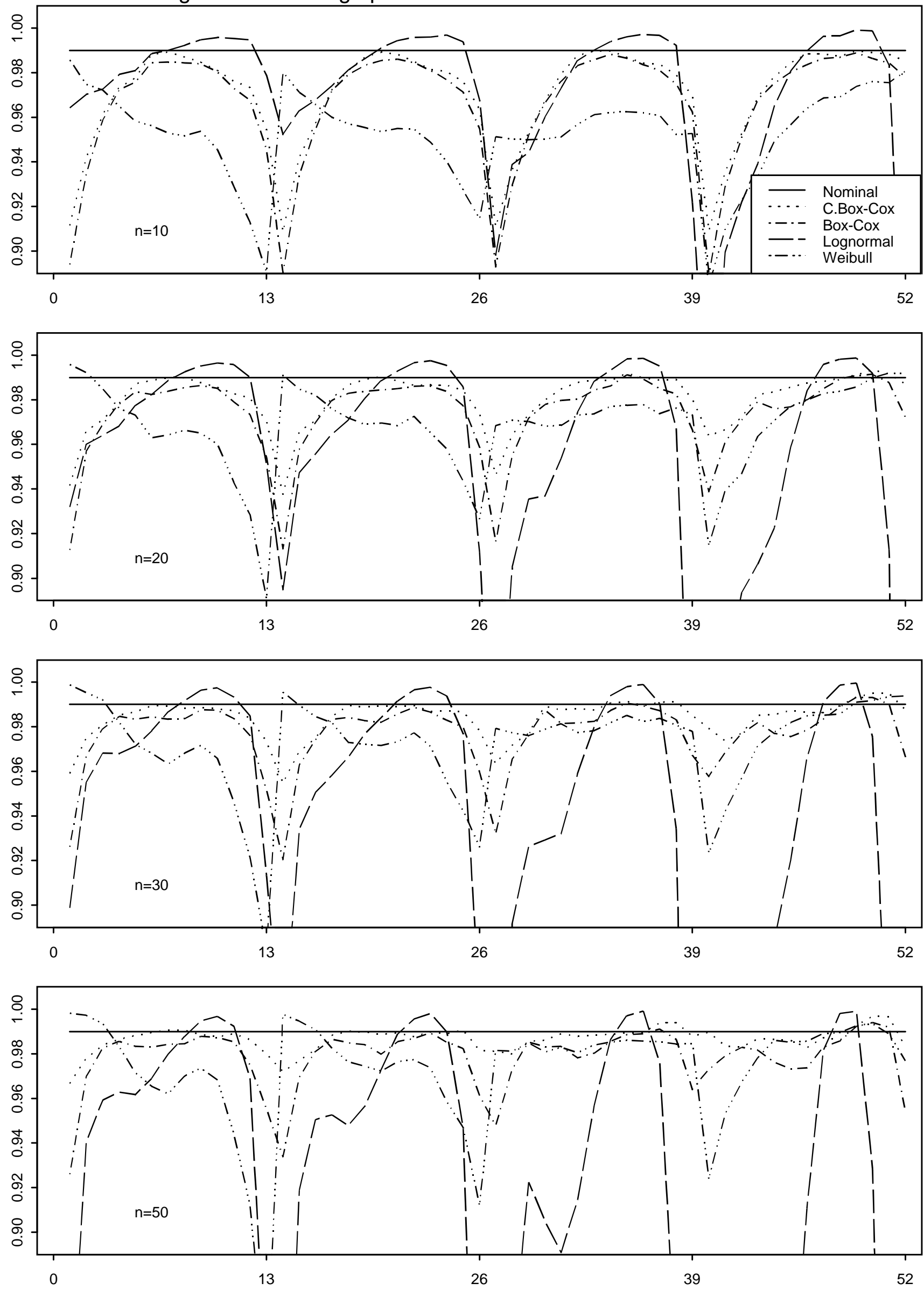

The horizontal index (1-52) corresponds to $\mathrm{p}=.01, .05, .1, .2, .3, .4, .5, .6, .7, .8, .9, .95, .99$, for each of beta=3, $2,1, .5$ 
Figure 4a. Coverage probabilities of the $90 \%$ : Inverse Gaussian data
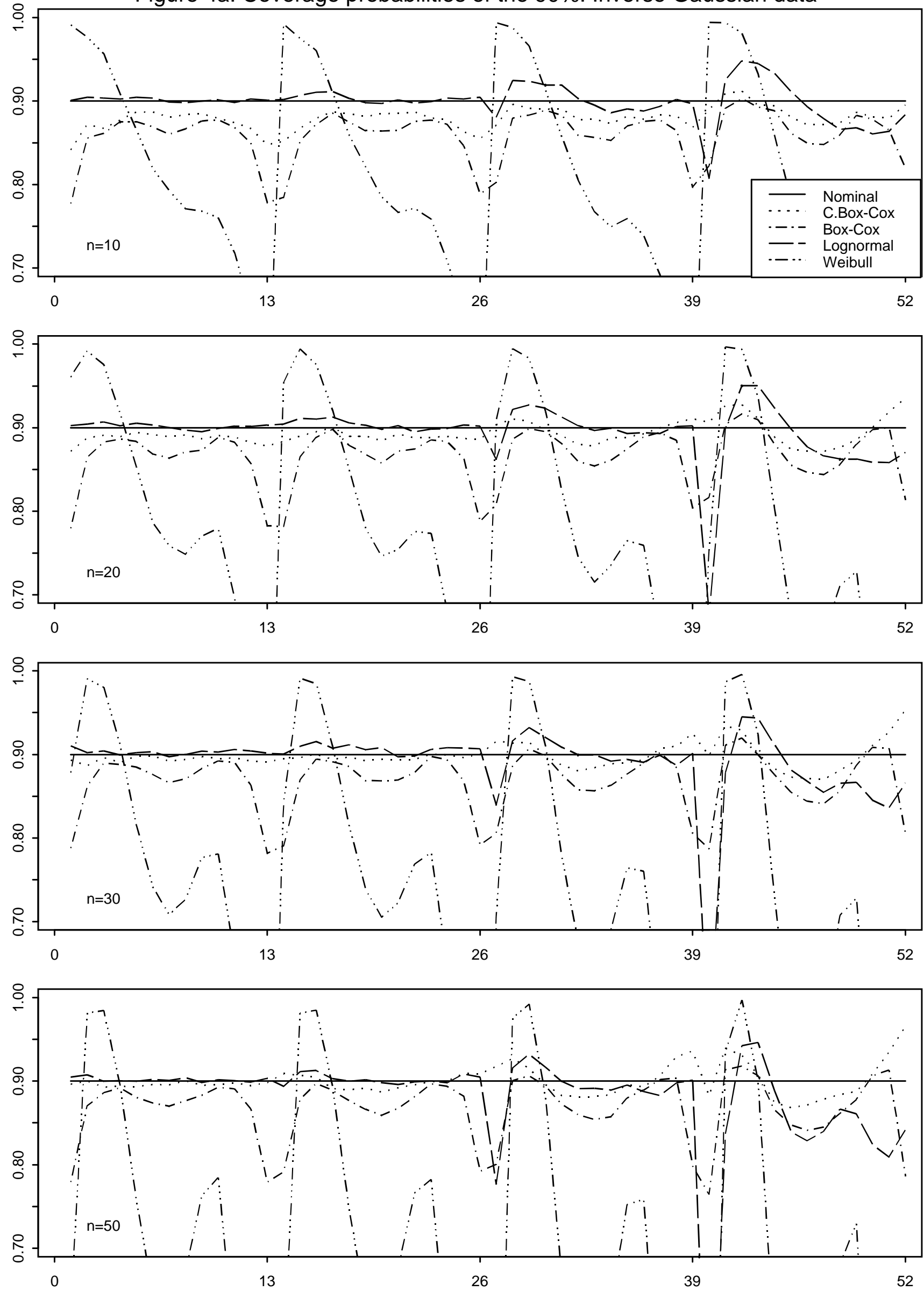

The horizontal index (1-52) corresponds to $\mathrm{p}=.01, .05, .1, .2, .3, .4, .5, .6, .7, .8, .9, .95, .99$, for each of beta $=9,4,1, .25$ 
Figure 4b. Coverage probabilities of the 95\% PLs: Inverse Gaussian data
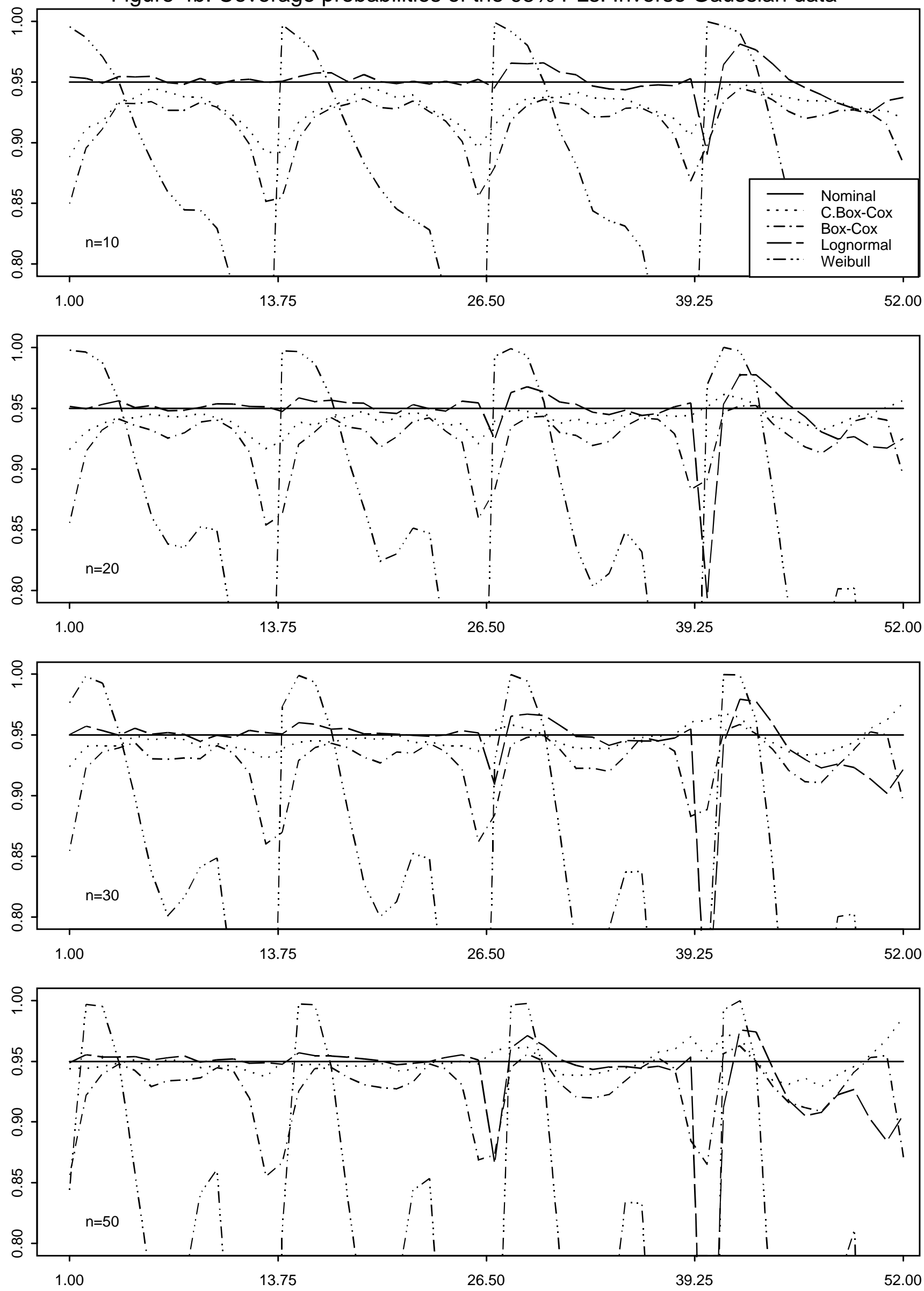

The horizontal index (1-52) corresponds to $\mathrm{p}=.01, .05, .1, .2, .3, .4, .5, .6, .7, .8, .9, .95, .99$, for each of beta=9, 4, 1, .25 
Figure 4c. Coverage probabilities of the $99 \%$ PLs: Inverse Gaussian data
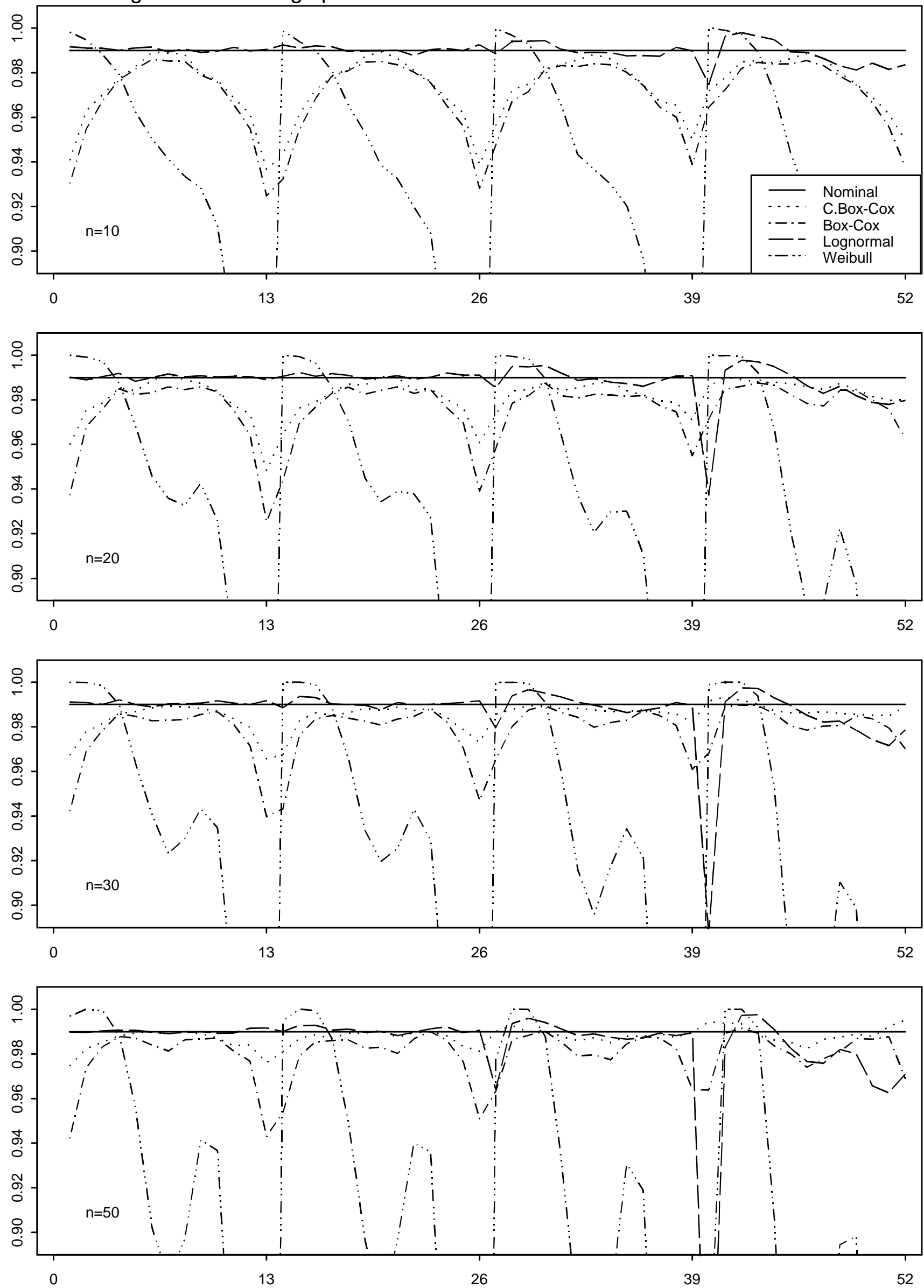

The horizontal index (1-52) corresponds to $\mathrm{p}=.01, .05, .1, .2, .3, .4, .5, .6, .7, .8, .9, .95, .99$, for each of beta=9, 4, $1, .25$ 
Figure 5. Percentile limits for strike duration data: 90\%(1st row), 95\%(2nd row), 99\%(3rd row)
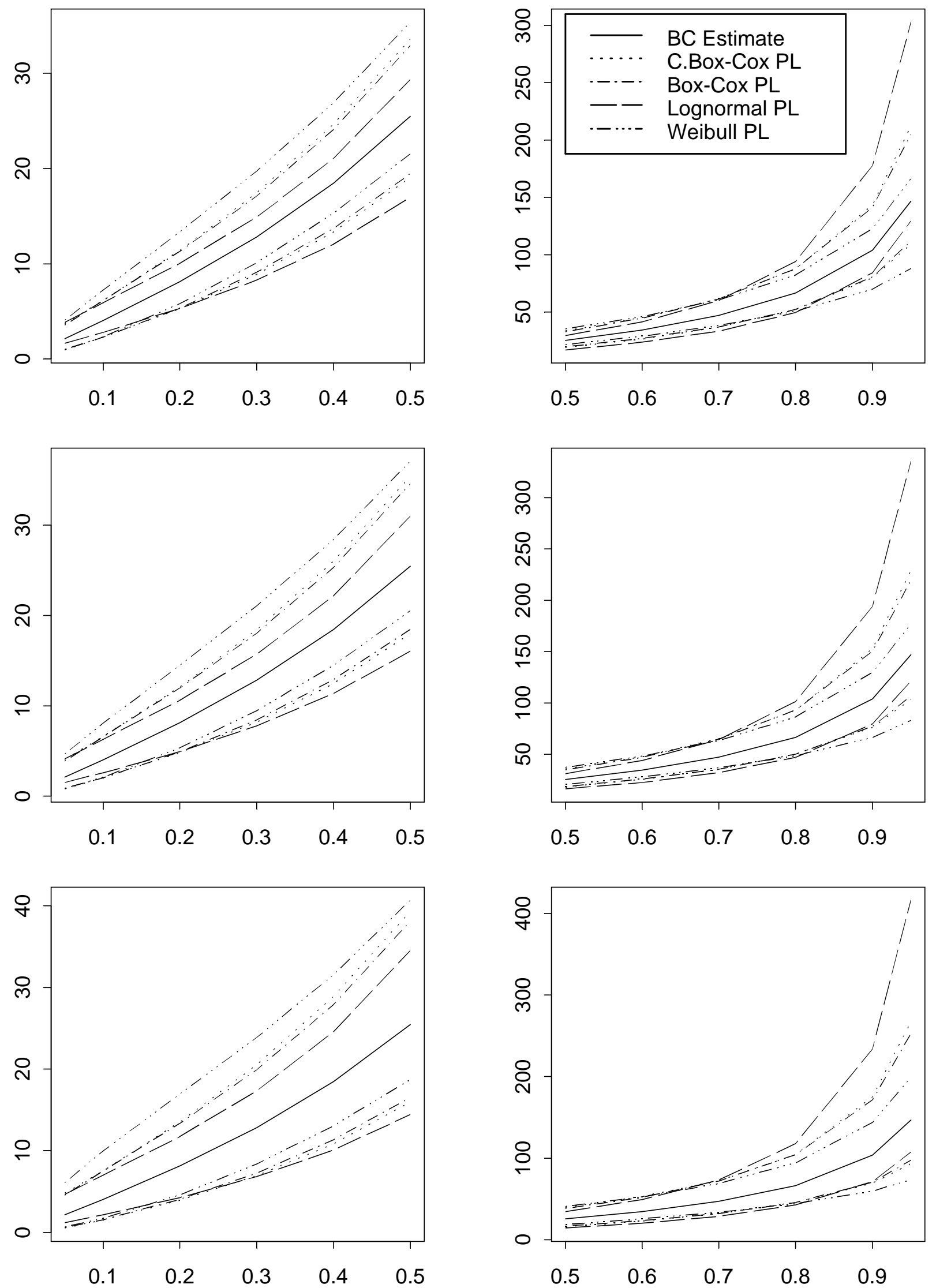